\title{
Robust Estimation and Inference for Threshold Models with Integrated Regressors
}

\author{
HAIQIANG CHEN* \\ Wang Yanan Institute for Studies in Economics, MOE Key Laboratory of Econometrics, \\ and Fujian Key Laboratory of Statistical Science, Xiamen University
}

July 24, 2013

\begin{abstract}
This paper studies the robust estimation and inference of threshold models with integrated regressors. We derive the asymptotic distribution of the profiled least squares (LS) estimator under the diminishing threshold effect assumption that the size of the threshold effect converges to zero. Depending on how rapidly this sequence converges, the model may be identified or only weakly identified and asymptotic theorems are developed for both cases. As the convergence rate is unknown in practice, a model-selection procedure is applied to determine the model identification strength and to construct robust confidence intervals, which have the correct asymptotic size irrespective of the magnitude of the threshold effect. The model is then generalized to incorporate endogeneity and serial correlation in error terms, under which, we design a Cochrane-Orcutt feasible generalized least squares (FGLS) estimator which enjoys efficiency gains and robustness against different error specifications, including both $I(0)$ and $I(1)$ errors. Based on this FGLS estimator, we further develop a sup-Wald statistic to test for the existence of the threshold effect. Monte Carlo simulations show that our estimators and test statistics perform well.
\end{abstract}

JEL Classification: C12, C22, C52

Keywords: Threshold effects; Integrated processes; Nonlinear cointegration; Weak identification.

\footnotetext{
${ }^{*}$ I would like to thank Professor Peter C.B. Phillips and the referees for helpful comments. Special thanks also go to Zongwu Cai, Jiti Gao, Wolfgang Härdle, Yongmiao Hong and seminar participants at Cornell University and the 3rd XMU-Humboldt Workshop. The research was supported in part by the National Nature Science Foundation of China grants \#11201390, \#71201137, \#71131008 and \#71271179, as well as Deutsche Forschungsgemeinschaft (DFG) through the SFB649 "Economic Risk". Address correspondence to Haiqiang Chen, Xiamen University, Xiamen, Fujian 361005, China; e-mail: hc335@xmu.edu.cn.
} 


\section{Introduction}

Threshold models, in which the model parameters switch when the value of a certain variable crosses a threshold, have received much attention over the past two decades. Given their ability to capture a very rich set of stylized facts of modern economics, such as multiple states, asymmetries and cyclical effects, the use of threshold models has been advocated in many empirical applications. Examples include the analysis of asymmetries in persistence in US output growth (Potter, 1995), nonlinearities in unemployment rates (Hansen, 1997), and multiple states in cross-country growth regressions (Durlauf and Johnson, 1995, Hansen, 2000), among numerous others. The estimation and asymptotics for variants of threshold models have been well established by Chan (1993), Hansen (1996, 2000), Gonzalo and Pitarakis (2002) and Seo and Linton (2007). More recently, Yu (2012) studies likelihood-based estimation and inference for a parametric discontinuous threshold regression model while Li and Ling (2012) study the least squares (LS) estimator of the multiple-regime threshold autoregressive (TAR) model.

However, most of the aforementioned studies maintain a restrictive assumption that the data should be either i.i.d. or stationary processes. Two exceptions are Caner and Hansen (2001) who study a threshold autoregressive model with a unit root and Gonzalo and Pitarakis (2006) who design a Wald statistic to test the threshold effect in a cointegrating regression. To the best of our knowledge, the asymptotic properties of threshold estimators, when all or some of the regressors are integrated processes, have not been established. In this paper, we investigate the robust estimation of threshold models with integrated regressors and establish its asymptotic properties allowing for different model identification strengths, endogenous regressors and serially correlated error terms, including both $\mathrm{I}(0)$ and $\mathrm{I}(1)$ errors.

Specifically, we consider a threshold model of the following form:

$$
y_{t}=\left\{\begin{array}{c}
\alpha_{1}^{\prime} x_{t}+u_{t}, \text { if } q_{t} \leq \gamma_{0} \\
\alpha_{2}^{\prime} x_{t}+u_{t}, \text { if } q_{t}>\gamma_{0}
\end{array},\right.
$$

where $x_{t}$ is a $d_{1}$-dimensional vector of integrated process of order $1(I(1))$ and $y_{t}$ is the dependent variable. ${ }^{1}$ The error term, which will be specified more precisely later, is denoted by $u_{t}$, and $q_{t}$ is the threshold variable. The threshold value $\gamma_{0} \in[\underline{\gamma}, \bar{\gamma}]$ is an unknown parameter pending estimation. If $u_{t}$ is a stationary process, Equation (1) can be regarded as a special case of nonlinear cointegration models, ${ }^{2}$ see Wang and Phillips (2009), Gao, King, Lu and Tjøstheim (2009a, 2009b), Bierens and Martins (2010) and Choi and Saikkonen (2010).

Equation (1) can be rewritten as

$$
y_{t}=\alpha^{\prime} x_{t}+\delta^{\prime} x_{t} I\left(q_{t} \leq \gamma_{0}\right)+u_{t},
$$

where $\alpha=\alpha_{2}$ and $\delta=\alpha_{1}-\alpha_{2}$. Here $I\left(q_{t} \leq \gamma_{0}\right)$ is an indicator function taking the value one if $q_{t} \leq \gamma_{0}$ and zero otherwise. The vector $(1,-\alpha)$ can be regarded as a benchmark long-run relationship between $y_{t}$ and $x_{t}$ and the 
term $\delta x_{t} I\left(q_{t} \leq \gamma_{0}\right)$ captures the deviation from the linear equilibrium. In the literature, $\delta$ is often assumed to be a deterministic sequence converging to zero at some rate as the sample size $n$ increases. This assumption is not only convenient for deriving the asymptotic distribution of threshold estimators as in Hansen (2000), but is also relevant in many empirical applications. For example, an important issue in the empirical finance literature is to investigate whether fundamental variables, such as the dividend-price ratio and the earning-price ratio, can predict asset returns. Linear prediction models have been extensively studied, but have failed to generate any unanimous conclusion (for more details, refer to Campbell and Yogo, 2006). ${ }^{3}$ Recently, Gonzalo and Pitarakis (2012) document a regime-specific predictability of S\&P 500 index returns from the dividend ratio based on a threshold model. However, as is well known, stock returns commonly behave as martingale differences, while fundamental variables are highly persistent (integrated or nearly integrated processes). This imbalance on the order of integration implies that such predictive relationship should be very weak, which happens if and only if $\alpha=0$ and $\delta$ is around zero in Equation (2). For more discussions about the issue of balance and localizing coefficients to zero in predictive regressions, one could refer to Phillips and Lee (2013).

In this paper, we follow the literature by assuming the size of threshold effect converges to zero. Depending on how rapidly this sequence converges, the threshold value $\gamma_{0}$ may be identified or only weakly identified and we develop asymptotic theorems for both cases. ${ }^{4}$ In the first case, we show that the profiled LS estimator is consistent and that its confidence intervals (CIs) can be constructed through inversion of certain standard test statistics; whereas in the second case, the profiled LS estimator is inconsistent and that its limiting distribution depends on some inestimable nuisance parameters. Because the standard method to construct CIs does not control the coverage probability, we take the supremum of quantiles for all possible values of nuisance parameters and then construct the least favorable CIs. These CIs have the correct asymptotic size under the weak identification case, but can be unnecessarily long when the model is identified. Motivated by Cheng (2008) and Shi and Phillips (2012), we then propose a model-selection procedure to choose the CIs. It can be shown that the CIs chosen by this method have approximately correct coverage probability irrespective of the magnitude of the threshold effect.

Furthermore, in a generalized model, we consider endogeneity and serial correlation, which are common in empirical studies with integrated regressors. Following Saikkonen (1991), we assume the error term to be an autoregressive (AR) process, and use leads and lags of innovations as extra regressors to deal with endogeneity. We then design a Cochrane-Orcutt feasible generalized least squares (FGLS) estimator to estimate the model. It is well known that, in linear cointegration models, the FGLS estimator can not improve the estimation efficiency, as Phillips and Park (1988) demonstrate by establishing their asymptotic equivalence. However, this equivalence does not hold when there exists a threshold effect and we analytically and numerically show that the FGLS estimation achieves more efficiency compared to the profiled LS estimation. The method to construct the robust CIs of the LS estimator can also be generalized for the FGLS estimator.

Another attraction of the FGLS estimator is its robustness with respect to different error specifications, including both $\mathrm{I}(0)$ and $\mathrm{I}(1)$ errors. This robustness allows us to design a sup-Wald statistic to test for the 
existence of threshold effects without any requirement on the stationarity of the regression error term and thus avoid the so-called joint hypothesis test problem in such models (see Balke and Fomby, 1997). The idea is similar to Perron and Yabu (2009), who consider testing for structural changes in the trend function of a time series using a quasi-FGLS procedure, without any requirement on whether the noise component is stationary or integrated. In our case, we use the sup-Wald test statistic based on the FGLS estimator to test for the existence of a threshold effect and the critical values are generated from a bootstrap method. Monte Carlo simulations show that the FGLS estimator and the test statistic perform very well, even the error is an I(1) process.

The remainder of the paper is organized as follows. Section 2 presents the basic model. Section 3 considers the estimator and its asymptotics for the basic model. Section 4 generalizes the basic model to allow for serially correlated errors and model endogeneity. Monte Carlo simulations are discussed in Section 5. Section 6 concludes the paper. All proofs are in the appendices.

Throughout the paper, $[n s]$ denotes the integer part of $n s$. The symbol ' $\stackrel{p}{\rightarrow}$ ' represents convergence in probability, ' $\Longrightarrow$ ' represents weak convergence, and $\stackrel{a . s .}{\rightarrow}$ represents almost sure convergence. All limits are defined as the sample size $n \rightarrow \infty$ unless otherwise stated. For a vector $x,\|\cdot\|$ signifies the Euclidean norm, i.e., $\|x\|=\sum x_{i}^{2}$. For a matrix $A,|A|=\left(\operatorname{tr}\left(A^{\prime} A\right)\right)^{1 / 2}$ denotes the Euclidean norm and $A^{\prime}$ denotes its transpose. $\int$ denotes integration from 0 to 1.

\section{The Basic Model}

The basic model we consider in the paper is given by

$$
y_{t}=\left\{\begin{array}{c}
\alpha_{1}^{\prime} x_{t}+u_{t}, \text { if } q_{t} \leq \gamma_{0} \\
\alpha_{2}^{\prime} x_{t}+u_{t}, \text { if } q_{t}>\gamma_{0}
\end{array},\right.
$$

where $x_{t}$ is a $d_{1}$-dimensional vector of unit root processes whose generating mechanism is given by

$$
x_{t}=x_{t-1}+v_{t}, t=1,2, \ldots n
$$

and we set $x_{0}=0$ for convenience, although $x_{0}=o_{a . s}(\sqrt{n})$ is sufficient for the asymptotic results.

For the component time series $u_{t}$ and $v_{t}$, we define the following partial sums as

$$
U_{n}(s) \equiv \frac{1}{\sqrt{n}} \sum_{t=1}^{[n s]} u_{t} \text {, and } V_{n}(s) \equiv \frac{1}{\sqrt{n}} \sum_{t=1}^{[n s]} v_{t}
$$

Before going further, we make the following assumptions.

Assumption 2.1. $\sup _{s \in[0,1]}\left\|\left(U_{n}(s), V_{n}(s)\right)-\left(B_{u}(s), \mathbf{B}_{v}(s)\right)\right\| \stackrel{\text { a.s. }}{\rightarrow} 0$ as $n \rightarrow \infty$, where $\left(B_{u}(s), \mathbf{B}_{v}(s)\right)$ is a vector of Brownian motions with a positive definite long-run covariance matrix.

Assumption 2.2. The error term $u_{t}$ satisfies the following three conditions: 
(a) $\mathbb{E}\left(u_{t}\right)=0, \mathbb{E}\left|u_{t}\right|^{4}<\infty ;$

(b) For each $n$, there exists a filtration $\digamma_{n, t}, t=1, \ldots, n$, such that $\left\{\left(u_{t}, \digamma_{n, t}\right): t \geq 1\right\}$ is a stationary and ergodic martingale difference sequence with $\mathbb{E}\left(u_{t}^{2} \mid \digamma_{n, t-1}\right)=\sigma_{u}^{2}<\infty$ almost surely for all $t=1, \ldots, n$;

(c) The term $\left(q_{t}, x_{t}\right)$ is adapted to the filtration $\digamma_{n, t-1}, t=1, \ldots, n$.

\section{Assumption 2.3.}

(a) The threshold parameter $\gamma_{0} \in[\underline{\gamma}, \bar{\gamma}]$;

(b) The threshold variable $q_{t}$ is strictly stationary, ergodic and $\alpha$-mixing with mixing coefficients $\alpha_{m}$ satisfying $\sum_{m=1}^{\infty} \alpha_{m}^{\frac{1}{2}-\frac{1}{r}}<\infty$ for $r>2$;

(c) $q_{t}$ has a continuous distribution $F(\cdot)$ and $f(\cdot)$ is the corresponding density function satisfying $0<f(\gamma) \leq$ $\bar{f}<\infty$ for all $\gamma \in[\underline{\gamma}, \bar{\gamma}]$.

Assumption 2.4. The $d_{1}$ dimensional $I(1)$ vector $x_{t}$ is not cointegrated.

Assumption (2.1) considers a strong approximation for $\left(U_{n}(s), V_{n}(s)\right)$, which is needed to derive the convergence rate of the threshold estimator. This assumption is stronger than the weak convergence result established by the multivariate invariance principle, but it is quite common in the literature, see Park and Phillips (2001), Wang and Phillips (2009) and Shi and Phillips (2012), among others. Sufficient conditions to derive the strong approximation are also well developed in the literature. For example, Park and Hahn (1999) establish conditions of strong approximations for general linear processes. Shao and Lu (1987) and Cai, Li and Park (2009) provide conditions of strong approximations for an $\alpha$-mixing process.

Assumption (2.2) supposes $\left\{\left(u_{t}, \digamma_{n, t}\right): t \geq 1\right\}$ to be a martingale difference sequence and $\left(q_{t}, x_{t}\right)$ is adapted to the filtration $\digamma_{n, t-1}, t=1, \ldots, n$. Under this assumption, $q_{t}$ and $x_{t}$ become predetermined given the filtration $\digamma_{n, t-1}$. One natural example of $\digamma_{t}$ is the $\sigma$-field generated by the information set $\left\{\left(u_{i}, q_{i+1}, x_{i+1}\right): 1 \leq i \leq\right.$ $t\}$. This assumption might be restrictive in linear cointegration models, but it is common in fully specified cointegrating regression models. It allows for arguments based on the martingale central limit theory to establish a weak convergence result for the empirical process $\sum_{t=1}^{[n s]} I_{t}\left(q_{t} \leq \gamma\right) u_{t}$ as in Caner and Hansen (2001). ${ }^{5}$ Assumption (2.2) does not allow for serially correlated errors and model endogeneity. However, in Section 4, we relax this assumption by adopting the so-called "leads and lags" approach or dynamic OLS estimator proposed by Saikkonen (1991).

Assumption (2.3) is very conventional in the literature of threshold models. The threshold variable $q_{t}$ is assumed to be strictly stationary and strong mixing for asymptotic purposes. The assumption excludes the case where $q_{t}$ is a unit root process, under which one may need to use another technique such as the triangular array asymptotics proposed by Andrews and McDermott (1995). Assumption (2.4) is standard in the literature of regressions with integrated processes. 
Following Caner and Hansen (2001), we define $W(s, \mu)$ as a two-parameter Brownian motion on $(s, \mu) \in[0,1]^{2}$, which is a zero-mean Gaussian process with covariance given by

$$
\mathbb{E}\left(W\left(s_{1}, \mu_{1}\right) W\left(s_{2}, \mu_{2}\right)\right)=\left(s_{1} \wedge s_{2}\right)\left(\mu_{1} \wedge \mu_{2}\right) .
$$

The two-parameter Brownian motion is a special tool to derive the limiting distribution in threshold models with integrated processes. Note that when $\mu=1$, we have $W(s, 1) \equiv W(s)$, which is a Brownian motion on $s \in[0,1]$.

Moreover, we can define the following stochastic integration with respect to $W(s, \mu)$ on the first argument $(s)$ while holding the second argument $(\mu)$ constant as

$$
\mathbf{J}_{1}(\mu)=\int \mathbf{B}_{v}(s) d W(s, \mu)=\operatorname{plim}_{n \rightarrow \infty} \sum_{t=1}^{n}\left(\mathbf{B}_{v}\left(\frac{t-1}{n}\right)\left(W\left(\frac{t}{n}, \mu\right)-W\left(\frac{t-1}{n}, \mu\right)\right)\right.
$$

where plim denotes convergence in probability and $\mathbf{J}_{1}(\mu)$ is a Gaussian process with an almost surely continuous sample path and the covariance kernel

$$
\mathbb{E}\left(\mathbf{J}_{1}\left(\mu_{1}\right) \mathbf{J}_{1}\left(\mu_{2}\right)\right)=\left(\mu_{1} \wedge \mu_{2}\right) \int \mathbf{B}_{v}(s) \mathbf{B}_{v}^{\prime}(s) d s
$$

\section{Main Results}

\subsection{Profiled LS Estimation and Asymptotics}

For ease of manipulation, we rewrite Equation (2) in a more compacted form:

$$
y_{t}=\theta^{\prime} A_{t}\left(\gamma_{0}\right)+u_{t},
$$

where $A_{t}\left(\gamma_{0}\right)=\left(x_{t}^{\prime}, x_{t}^{\prime}\left(\gamma_{0}\right)\right)^{\prime}, x_{t}\left(\gamma_{0}\right)=x_{t} I\left(q_{t} \leq \gamma_{0}\right)$, and $\theta=\left(\alpha^{\prime}, \delta^{\prime}\right)^{\prime}$.

For any fixed $\gamma \in[\underline{\gamma}, \bar{\gamma}]$, the following model is estimated:

$$
y_{t}=\widehat{\theta}(\gamma)^{\prime} A_{t}(\gamma)+\widehat{u}_{t}(\gamma),
$$

where $\widehat{\theta}(\gamma)$ is given by

$$
\widehat{\theta}(\gamma)=\left[\sum_{t=1}^{n} A_{t}(\gamma) A_{t}(\gamma)^{\prime}\right]^{-1}\left[\sum_{t=1}^{n} A_{t}(\gamma) y_{t}\right] .
$$

The sum of squared residuals is defined as

$$
S S R_{n}(\gamma)=\sum_{t=1}^{n} \widehat{u}_{t}(\gamma)^{2}=\sum_{t=1}^{n}\left(y_{t}-\widehat{\theta}(\gamma)^{\prime} A_{t}(\gamma)\right)^{2},
$$


and we define the profiled LS estimator of $\gamma_{0}$ as the value that minimizes $S S R_{n}(\gamma)$, i.e.,

$$
\widehat{\gamma}_{n}=\arg \min _{\gamma \in[\underline{\gamma}, \bar{\gamma}]} S S R_{n}(\gamma)
$$

The term $S S R_{n}(\gamma)$ is not differentiable due to the presence of the indicator functions; thus, we can not write $\widehat{\gamma}_{n}$ in closed form from first-order conditions. Following Hansen (2000), we adopt a grid-searching method. Specifically, we divide $[\underline{\gamma}, \bar{\gamma}]$ into $N$ quantiles and let $\Gamma_{N}=\left\{q_{1}, q_{2}, \ldots q_{N}\right\}$. The estimator $\widehat{\gamma}_{N}=\arg \min _{\gamma \in \Gamma_{N}} S S R_{n}(\gamma)$ is a good approximation to $\widehat{\gamma}_{n}$ when $N$ is large enough. The other parameters are then estimated by using the point estimate $\widehat{\gamma}_{n}$ via $\widehat{\theta}=\widehat{\theta}\left(\widehat{\gamma}_{n}\right)$ and $\widehat{\sigma}_{u}^{2}=\frac{1}{n} \sum_{t=1}^{n} \widehat{u}_{t}\left(\widehat{\gamma}_{n}\right)^{2}$.

In what follows, we set $\delta=\delta_{n}=n^{-1 / 2-\tau} \delta_{0}$, where $-1 / 2<\tau \leq 1 / 2$ and $\delta_{0} \in R$ is a fixed parameter. Under this assumption, the size of the threshold effect converges to zero with the rate $n^{-1 / 2-\tau}$, where the value of $\tau$ determines the identification strength of the threshold value $\gamma_{0}$. We exclude the case with $\tau>1 / 2$ since the nonlinear term is negligible asymptotically. In addition, when $\tau<-1 / 2$, the nonlinear term is explosive and is also excluded. We also exclude the case of $\tau=-1 / 2$ to focus on the limiting behavior of $\widehat{\gamma}_{n}$ when $\delta_{n} \rightarrow 0$.

The following theorem states the limiting results for $\widehat{\gamma}_{n}$.

Theorem 3.1 Under Assumptions (2.1) - (2.4) and $\delta_{n}=n^{-1 / 2-\tau} \delta_{0}$, the following limiting results hold:

Case 1: if $-1 / 2<\tau<1 / 2$, then

$$
n^{1-2 \tau}\left|\widehat{\gamma}_{n}-\gamma_{0}\right|=O_{p}(1)
$$

Furthermore,

$$
n^{1-2 \tau} \lambda\left(\widehat{\gamma}-\gamma_{0}\right)=r^{*} \Rightarrow \arg \max _{r \in(-\infty, \infty)}\left(\Lambda(r)-\frac{1}{2}|r|\right),
$$

where

$$
\lambda=\frac{\delta_{0}^{\prime}\left(\int \mathbf{B}_{v}(s) \mathbf{B}_{v}^{\prime}(s) d s\right) \delta_{0} f_{0}}{\sigma_{u}^{2}}
$$

with $f_{0}=f\left(\gamma_{0}\right)$ and $\Lambda(r)$ is a two-sided Brownian motion on the real line defined as:

$$
\Lambda(r)=\left\{\begin{array}{c}
\Lambda_{1}(r) \text { if } r>0 \\
0 \quad \text { if } r=0 \\
\Lambda_{2}(-r) \text { if } r<0
\end{array} .\right.
$$

The processes $\Lambda_{1}(r)$ and $\Lambda_{2}(r)$ are two independent standard Brownian motions on $[0, \infty)$. 
Case 2: if $\tau=1 / 2$, then $\widehat{\gamma}_{n} \Rightarrow \gamma\left(\gamma_{0}, \delta_{0}\right)$ and $\gamma\left(\gamma_{0}, \delta_{0}\right)$ is a random variable that maximizes $Q\left(\gamma, \gamma_{0}, \delta_{0}\right)$, where

$$
Q\left(\gamma, \gamma_{0}, \delta_{0}\right)=\frac{1}{F(\gamma)(1-F(\gamma))} \Gamma_{1}^{\prime}(\gamma)\left(\int \mathbf{B}_{v}(s) \mathbf{B}_{v}^{\prime}(s) d s\right)^{-1} \Gamma_{1}(\gamma)
$$

with

$$
\Gamma_{1}(\gamma)=\Gamma(\gamma)+\left[F\left(\gamma \wedge \gamma_{0}\right)-F(\gamma) F\left(\gamma_{0}\right)\right]\left(\int \mathbf{B}_{v}(s) \mathbf{B}_{v}^{\prime}(s) d s\right) \delta_{0}
$$

and

$$
\Gamma(\gamma)=\sigma_{u} \int \mathbf{B}_{v}(s) d[W(s, F(\gamma))-F(\gamma) W(s)]
$$

Theorem 3.1 shows that the convergence results for $\widehat{\gamma}_{n}$ depend critically on the value of $\tau$, which characterizes the convergence speed of $\delta_{n} \cdot{ }^{6}$

If $-1 / 2<\tau<1 / 2$, the threshold effect is identifiable and $\widehat{\gamma}_{n}$ is a consistent estimator. The rate of convergence is $n^{1-2 \tau}$, which is decreasing in $\tau$. The limiting distribution of $\widehat{\gamma}_{n}$ has the same form as that found for the stationary threshold model in Hansen (2000), but the scale factor $\lambda$ is different. Note that $f_{0}$ is the density of $q_{t}$ at $\gamma_{0}$. Intuitively, a larger $f_{0}$ implies more data points around $\gamma_{0}$, making $\widehat{\gamma}$ more accurate.

To generate the confidence interval of $\gamma$, we invert the following likelihood ratio statistic for the null hypothesis $\gamma=\gamma_{0}$, given by

$$
L R_{n}\left(\gamma_{0}\right)=n \frac{S S R_{n}\left(\gamma_{0}\right)-S S R_{n}\left(\widehat{\gamma}_{n}\right)}{S S R_{n}\left(\widehat{\gamma}_{n}\right)}
$$

where $\widehat{\gamma}_{n}$ is the profiled LS estimator. Using a similar argument of Theorem 2 in Hansen (2000), we can show that $L R_{n}\left(\gamma_{0}\right) \Rightarrow \sup _{r \in(-\infty, \infty)}(2 \Lambda(r)-|r|)$. Denote $q_{\gamma, 1-a}^{I}$ as the $1-a$ quantile of the random variable $\sup _{r \in(-\infty, \infty)}(2 \Lambda(r)-|r|)$ and $q_{\gamma, 1-a}^{I}$ can be calculated by the formula $q_{\gamma, 1-a}^{I}=-2 \ln (1-\sqrt{1-a})$. Thus, the $a$-level confidence interval of $\gamma$ in the case of identification can be expressed as

$$
C I_{\gamma, n}^{I}(\alpha)=\left\{\gamma: L R_{n}(\gamma) \leq q_{\gamma, 1-a}^{I}\right\}
$$

If $\tau=1 / 2$, the threshold effect is only weakly identified. The profiled LS estimator $\widehat{\gamma}_{n}$ converges to a random variable $\gamma\left(\gamma_{0}, \delta_{0}\right)$, reflecting the lack of information. Since $\gamma_{0}$ and $\delta_{0}$ are not estimable, any statistical inference based on them is impossible. Following Cheng (2008) and Shi and Phillips (2012), we define the least favorable CI which is large enough for all possible $\gamma_{0}$ and $\delta_{0}$. Denote $q_{\gamma, 1-a}^{W}\left(\gamma_{0}, \delta_{0}\right)$ as the $1-a$ quantile of $\left|\gamma\left(\gamma_{0}, \delta_{0}\right)-\gamma_{0}\right|$ for each $\gamma_{0} \in[\underline{\gamma}, \bar{\gamma}]$ and $\delta_{0} \in R$. The $a$-level CI, given $\gamma_{0}$ and $\delta_{0}$, is then defined as

$$
C I_{\gamma, n}^{W}\left(1-a, \gamma_{0}, \delta_{0}\right)=\left\{\gamma:\left|\widehat{\gamma}_{n}-\gamma\right| \leq q_{\gamma, 1-a}^{W}\left(\gamma_{0}, \delta_{0}\right)\right\}
$$


Since $\gamma_{0}$ and $\delta_{0}$ are two unknown variables, we define a robust quantile by taking the supremum of all possible $\gamma_{0}$ and $\delta_{0}$. Let

$$
q_{\gamma, 1-a}^{W}=\sup _{\gamma_{0} \in[\underline{\gamma}, \bar{\gamma}]} \sup _{\delta_{0} \in R} q_{\gamma, 1-a}^{W}\left(\gamma_{0}, \delta_{0}\right)
$$

The $a$-level least favorable CI in the case of weak identification is then defined as

$$
C I_{\gamma, n}^{W}(a)=\left\{\gamma:\left|\widehat{\gamma}_{n}-\gamma\right| \leq q_{\gamma, 1-a}^{W}\right\}
$$

\subsection{Robust Confidence Interval}

In empirical studies, $\tau$ is unknown, raising the question of which CI should be used. In this subsection, based on a model selection procedure, we construct a robust CI which has approximately correct coverage probability irrespective of the value of $\tau$.

For a fixed $\gamma \in[\underline{\gamma}, \bar{\gamma}]$, let $X(\gamma)=\left(x_{1}(\gamma), x_{2}(\gamma), \ldots, x_{n}(\gamma)\right)^{\prime}$ and $X=\left(x_{1}, x_{2}, \ldots, x_{n}\right)^{\prime}$. The Wald test statistic for testing $H_{0}: \delta_{n}=0$ can be defined as

$$
T_{n}(\gamma)=\widehat{\delta}_{n}(\gamma)^{\prime}\left(X^{\prime}(\gamma)\left(I-P_{n}\right) X(\gamma)\right) \widehat{\delta}_{n}(\gamma) / \widehat{\sigma}_{u}^{2}
$$

where $P_{n}$ is the projection matrix of $X$, given by $P_{n}=X\left(X^{\prime} X\right)^{-1} X^{\prime}$. We define the sup-Wald test statistic as

$$
T_{n}=\sup _{\gamma \in[\underline{\gamma}, \bar{\gamma}]} T_{n}(\gamma)
$$

The following theorem explores the limiting behaviors of the sup-Wald statistic under different model identification strengths.

TheOrem 3.2 Under Assumptions (2.1)-(2.4) and $\delta_{n}=n^{-1 / 2-\tau} \delta_{0}$, where $\delta_{0}$ is a nonzero constant, the following limiting results hold:

(i) if $-1 / 2<\tau<1 / 2$, then $T_{n} \stackrel{p}{\rightarrow} \infty$.

(ii) if $\tau=1 / 2$, then

$$
T_{n} \Rightarrow T_{1}=\sup _{\gamma \in[\underline{\gamma}, \bar{\gamma}]} T_{1}(\gamma)=\sup _{\gamma \in[\underline{\gamma}, \bar{\gamma}]} \frac{1}{\sigma_{u}^{2}(F(\gamma)(1-F(\gamma))} \Gamma_{1}(\gamma)^{\prime}\left(\int \mathbf{B}_{v}(s) \mathbf{B}_{v}^{\prime}(s) d s\right)^{-1} \Gamma_{1}(\gamma)
$$

where $\Gamma_{1}(\gamma)$ is defined by Equation (9).

Theorem 3.2 shows that $T_{n} \stackrel{p}{\rightarrow} \infty$ if $-1 / 2<\tau<1 / 2$ and $T_{n}<\infty$ if $\tau=1 / 2$. This result enables us to develop the following model selection procedure. We define $\left\{\kappa_{n}: n \geq 1\right\}$ as a sequence of constants that diverge to infinity as $n \rightarrow \infty$. The parameter $\kappa_{n}$ is referred to as a tuning parameter and we require the sequence $\kappa_{n}$ to diverge to infinity at a rate slower than $n^{\nu}$ for any $\nu>0$, i.e., $n^{-\nu} \kappa_{n}^{1 / 2} \rightarrow 0$. Suitable choices of $\kappa_{n}$ include 
$d(\ln (n))^{2}$ where $d$ is a positive constant, in accordance with $B I C$. The model selection procedure is designed to choose the model with the identified threshold effect if $T_{n}>\kappa_{n}$ and to choose the model with the weakly identified threshold effect otherwise. We use the CI chosen through this procedure as the final CI.

Specifically, for each confidence level $a$, define

$$
C I_{\gamma, n}(a)=\left\{\begin{array}{l}
C I_{\gamma, n}^{I}(a), \text { if } T_{n}>\kappa_{n} \\
C I_{\gamma, n}^{W}(a), \text { if } T_{n} \leq \kappa_{n}
\end{array}\right\} .
$$

We focus on the smallest finite sample coverage probability of $C I_{\gamma, n}(a)$ over the whole parameter space, which can be approximated by the following asymptotic size

$$
\operatorname{AsyS} Z_{\gamma}(a)=\lim \inf _{n \rightarrow \infty} \inf _{\delta_{0} \in R} \inf _{\gamma \in[\underline{\gamma}, \bar{\gamma}]} \operatorname{Pr}\left(\gamma \in C I_{\gamma, n}(a)\right)
$$

The following theorem shows that the robust CI has the correct asymptotic size.

Theorem 3.3 Under Assumptions (2.1) - (2.4), for any $a \in(0,1)$, we have AsySZ $Z_{\gamma}(a)=a$.

\section{The Generalized Model}

In many economic applications of cointegration, error terms are serially correlated and correlated with regressors. Under these conditions, it is well known that the ordinary least squares (OLS) estimator contains a second-order bias in linear cointegration models. Several efficient estimators have been proposed, such as the fully modified (FM) OLS estimator of Phillips and Hansen (1990), the canonical cointegrating regressions (CCR) estimator of Park (1992) and the dynamic ordinary least squares (DOLS) estimator proposed by Saikkonen (1991). In the following, we generalize the basic model in Section 2 to allow for serial correlation and model endogeneity.

More formally, we introduce the following assumptions.

Assumption 4.1. In Equation (1), $u_{t}$ can be decomposed as

$$
\begin{aligned}
& u_{t}=\sum_{i=1}^{d_{1}} \sum_{j=-K}^{K} \beta_{i j} v_{i, t-j}+\eta_{t}=\beta^{\prime} z_{t}+\eta_{t}, \\
& \eta_{t}=\rho \eta_{t-1}+\varepsilon_{t}, \text { with } \rho \in(-1,1],
\end{aligned}
$$

where $z_{t}=\left(v_{1, t-K}, \ldots, v_{d_{1}, t+K}\right)$ and $\beta=\left(\beta_{1,-K}, \ldots, \beta_{d_{1}, K}\right)$. The error term $\varepsilon_{t}$ satisfies the following conditions:

(a) $\mathbb{E}\left(\varepsilon_{t}\right)=0, \mathbb{E}\left|\varepsilon_{t}\right|^{4}<\infty ;$

(b) For each $n$, there exists a filtration $\digamma_{n, t}, t=1, \ldots, n$, such that $\left\{\left(\varepsilon_{t}, \digamma_{n, t}\right): t \geq 1\right\}$ is a stationary and ergodic martingale difference sequence with $\mathbb{E}\left(\varepsilon_{t}^{2} \mid \digamma_{n, t-1}\right)=\sigma^{2}<\infty$ almost surely for all $t=1, \ldots, n$;

(c) The term $\left(q_{t}, x_{t}\right)$ is adapted to the filtration $\digamma_{n, t-1}, t=1, \ldots, n$. 
Assumption 4.2. Let $\xi_{n}(s) \equiv \frac{1}{\sqrt{n}} \sum_{t=1}^{[n s]} \varepsilon_{t}$ and $\sup _{s \in[0,1]}\left\|\left(\xi_{n}(s), V_{n}(s)\right)-\left(B_{\varepsilon}(s), \mathbf{B}_{v}(s)\right)\right\| \stackrel{a . s .}{\rightarrow} 0$ as $n \rightarrow \infty$, where $\left(B_{\varepsilon}(s), \mathbf{B}_{v}(s)\right)$ is a vector of Brownian motions with a positive definite long-run covariance matrix.

Under Assumption (4.1), the model endogeneity can be fully captured by $\beta^{\prime} z_{t}$, where $z_{t}$ is a vector of leads and lags of $\Delta x_{t}$. The parameter $K$ can diverge to infinity as the sample size increases. The idea of using leads and lags to deal with endogeneity in cointegration models was proposed by Saikkonen (1991). We assume $\beta$ remains constant to focus on the threshold effect occurring in the cointegrating relationship. The extension allowing $\beta$ to be regime-sensitive would be interesting and is left to future study. The term $\eta_{t}$ is assumed to be $A R(1)$ and $\rho$ controls the stationarity of $\eta_{t}$. If $\rho=1, \eta_{t}$ is a unit root process and the model describes a structural spurious relationship, ${ }^{7}$ while if $\rho<1, \eta_{t}$ is a stationary process and the model is a cointegrating relationship.

To estimate a regression with serial correlation, the Cochrane-Orcutt FGLS procedure is usually adopted. In linear cointegration models, as shown in Phillips and Park (1988), the FGLS estimator and the OLS estimator are equivalent in asymptotics. The Cochrane-Orcutt FGLS estimator also works for spurious regressions, as Phillips and Hodgson (1994) demonstrate by proving the asymptotic equivalence of the FGLS estimator to the OLS estimator when the error is an I(1) process. However, in the presence of threshold effects, there is no asymptotic equivalence between FGLS and OLS estimators. The following simple sketch may help to illustrate this difference.

For a linear cointegrating regression after transformation, $y_{t}-\rho y_{t-1}=\alpha^{\prime}\left(x_{t}-\rho x_{t-1}\right)+\left(\eta_{t}-\rho \eta_{t-1}\right)$, and

$$
\begin{aligned}
n\left(\widehat{\alpha}_{F G L S}-\alpha\right) & =\left(\sum_{t=1}^{n}\left(x_{t}-\rho x_{t-1}\right)\left(x_{t}-\rho x_{t-1}\right)^{\prime}\right)^{-1}\left(\sum_{t=1}^{n}\left(x_{t}-\rho x_{t-1}\right)\left(\eta_{t}-\rho \eta_{t-1}\right)\right) \\
& \Rightarrow\left(\int \mathbf{B}_{v}(s) \mathbf{B}_{v}^{\prime}(s) d s\right)^{-1} \int \mathbf{B}_{v}(s) d B_{\eta}(s),
\end{aligned}
$$

which is the same as the limiting result of the OLS estimator. However, for a cointegrating regression with a threshold effect after transformation, $y_{t}-\rho y_{t-1}=\alpha^{\prime}\left(x_{t}-\rho x_{t-1}\right)+\delta_{n}^{\prime}\left(x_{t}(\gamma)-\rho x_{t-1}(\gamma)\right)+\left(\eta_{t}-\rho \eta_{t-1}\right)=$ $\alpha^{\prime} \widetilde{x}_{t}+\delta_{n}^{\prime} \widetilde{x}_{t}(\gamma)+\left(\eta_{t}-\rho \eta_{t-1}\right)$, and $\rho$ can not be canceled in the limiting result because the denominator $\sum_{t=1}^{n} \widetilde{x}_{t}(\gamma) \widetilde{x}_{t}(\gamma)^{\prime} \Rightarrow\left(\left(1+\rho^{2}\right) F(\gamma)-2 \rho F_{1}(\gamma, \gamma)\right) \int \mathbf{B}_{v}(s) \mathbf{B}_{v}^{\prime}(s) d s$, which depends on $\rho$, the marginal distribution function $F(\gamma) \equiv \operatorname{Pr}\left(q_{t} \leq \gamma\right)$ and the joint distribution $F_{1}(\gamma, \gamma) \equiv \operatorname{Pr}\left(q_{t} \leq \gamma, q_{t-1} \leq \gamma\right)$, whereas the nominator $\sum_{t=1}^{n} \widetilde{x}_{t}(\gamma)\left(\eta_{t}-\rho \eta_{t-1}\right) \Rightarrow\left(1-\rho^{2}\right) \int \mathbf{B}_{v}(s) d B_{\eta}(s)$.

\subsection{FGLS Estimator}

To obtain a feasible GLS estimator, we first estimate the threshold value $\gamma_{0}$ through the profiled LS estimator without considering serial correlation and endogeneity. Then we estimate $\widehat{\rho}$ from the estimated error terms. Finally, we construct the Cochrane-Orcutt FGLS estimator based on $\widehat{\rho}$. 
Specifically, we estimate $\gamma_{0}$ using

$$
\widehat{\gamma}_{n}=\arg \min _{\gamma \in[\underline{\gamma}, \bar{\gamma}]} S S R_{n}(\gamma)
$$

where $S S R_{n}(\gamma)$ is the sum of squared residuals for the regression

$$
y_{t}=\widehat{\alpha}^{\prime} x_{t}+\widehat{\delta}_{n}^{\prime} x_{t} I\left(q_{t} \leq \gamma\right)+\widehat{\beta}^{\prime} z_{t}+\widehat{\eta}_{t}
$$

By estimating the $A R$ model

$$
\widehat{\eta}_{t}\left(\widehat{\gamma}_{n}\right)=\widehat{\rho}_{t-1}\left(\widehat{\gamma}_{n}\right)+\widehat{\varepsilon}_{t}
$$

the OLS estimator $\widehat{\rho}$ is estimated, where $\widehat{\eta}_{t}\left(\widehat{\gamma}_{n}\right)=y_{t}-\widehat{\alpha}^{\prime}\left(\widehat{\gamma}_{n}\right) x_{t}-\widehat{\delta}_{n}^{\prime}\left(\widehat{\gamma}_{n}\right) x_{t} I\left(q_{t} \leq \widehat{\gamma}_{n}\right)-\widehat{\beta}^{\prime}\left(\widehat{\gamma}_{n}\right) z_{t}$.

The following theorem establishes the consistency and the convergence rate of $\widehat{\rho}$.

Theorem 4.1 Under Assumptions (4.1), (4.2), (2.3) and (2.4), $\widehat{\rho} \rightarrow \rho$ as $n \rightarrow \infty$. Furthermore, $|\widehat{\rho}-\rho|=$ $O_{p}\left(n^{-1 / 2}\right)$ if $\rho<1$, and $|\widehat{\rho}-1|=O_{p}\left(n^{-1}\right)$ if $\rho=1$.

Theorem 4.1 shows that $\widehat{\rho}$ is consistent even when $\rho=1$, i.e., the regression is a spurious relationship. The convergence rates are different due to the different convergence speeds of integrated and stationary processes. Moreover, we find that the limiting behavior of $\widehat{\rho}$ is not affected by the identification strength of the threshold effect. The intuition is as follows. If $\tau<1 / 2, \gamma_{0}$ can be consistently estimated by $\widehat{\gamma}_{n}$. In each regime, if $\rho<1$, the coefficients can be consistently estimated as well and thus it is obvious that $\widehat{\rho} \stackrel{p}{\rightarrow} \rho$. If $\rho=1$, the coefficient estimators are not consistent; however, this inconsistency implies that the residual term $\widehat{\eta}_{t}\left(\widehat{\gamma}_{n}\right)$ is a unit root and that $\widehat{\rho} \stackrel{p}{\rightarrow} \rho=1$. If $\tau=1 / 2, \widehat{\gamma}_{n}$ is not consistent as shown in Theorem 3.1; however, the nonlinear term $\widehat{\delta}_{n}^{\prime} x_{t} I\left(q_{t} \leq \gamma\right)$ decays to zero so fast that it has no impact on the estimation of $\rho$ asymptotically. Following Choi et al. (2008), the consistency of $\widehat{\rho}$ can also be obtained.

Based on the consistent estimator $\widehat{\rho}$, we construct the following Cochrane-Orcutt FGLS estimators. We first define $\widetilde{y}_{t}=y_{t}-\widehat{\rho} y_{t-1}$, and $\widetilde{z}_{t}, \widetilde{x}_{t}, \widetilde{\eta}_{t}$ in the same way. For each $\gamma \in[\underline{\gamma}, \bar{\gamma}]$, define

$$
\widetilde{x}_{t}(\gamma)=x_{t} I\left(q_{t} \leq \gamma\right)-\widehat{\rho} x_{t-1} I\left(q_{t-1} \leq \gamma\right)
$$

Let $\widetilde{A}_{1 t}(\gamma)=\left(\widetilde{x}_{t}^{\prime}, \widetilde{x}_{t}(\gamma)^{\prime}, \widetilde{z}_{t}^{\prime}\right)^{\prime}$ and stack $\widetilde{x}_{t}, \widetilde{y}_{t}, \widetilde{z}_{t}, \widetilde{x}_{t}(\gamma)$ and $\widetilde{A}_{1 t}(\gamma)$ to get the matrices: $\widetilde{X}, \widetilde{Y}, \widetilde{Z}, \widetilde{X}(\gamma)$ and $\widetilde{A}_{1}(\gamma)$.

After the transformation,

$$
\widetilde{y}_{t}=\alpha^{\prime} \widetilde{x}_{t}+\delta_{n}^{\prime} \widetilde{x}_{t}(\gamma)+\beta^{\prime} \widetilde{z}_{t}+\widetilde{\eta}_{t}=\widetilde{\theta}^{\prime} \widetilde{A}_{1 t}(\gamma)+\widetilde{\eta}_{t}
$$


Using Equation (20), for each $\gamma$, we can define

$$
\widehat{\widetilde{\theta}}(\gamma)=\left[\sum_{t=2}^{n} \widetilde{A}_{1 t}(\gamma) \widetilde{A}_{1 t}(\gamma)^{\prime}\right]^{-1}\left[\sum_{t=2}^{n} \widetilde{A}_{1 t}(\gamma) \widetilde{y}_{t}\right]
$$

and the FGLS threshold estimator can be defined as

$$
\widetilde{\gamma}_{n}=\arg \min _{\gamma \in[\underline{\gamma}, \bar{\gamma}]}\left(\widetilde{S S R}_{n}(\gamma)\right)
$$

where $\widetilde{S S R}_{n}(\gamma)$ is the sum of squared residuals given by

$$
\widetilde{S S R}_{n}(\gamma)=\sum_{t=2}^{n}\left(\widetilde{y}_{t}(\gamma)-\widehat{\widetilde{\theta}}(\gamma)^{\prime} \widetilde{A}_{1 t}(\gamma)\right)^{2}
$$

The following theorem establishes the limiting results of the FGLS estimator.

THeOREm 4.2 Under Assumptions (4.1), (4.2), (2.3) and (2.4), the following results hold:

Case 1: if $-1 / 2<\tau<1 / 2$, then $n^{1-2 \tau}\left|\widetilde{\gamma}_{n}-\gamma_{0}\right|=O_{p}(1)$. Furthermore,

$$
n^{1-2 \tau} \widetilde{\lambda}\left(\widetilde{\gamma}_{n}-\gamma_{0}\right)=r^{*} \Rightarrow \arg \max _{r \in(-\infty, \infty)}\left(\Lambda(r)-\frac{1}{2}|r|\right)
$$

where

$$
\widetilde{\lambda}=\frac{\left(1+\rho^{2}\right)\left(\delta_{0}^{\prime} \int \mathbf{B}_{v}(s) \mathbf{B}_{v}^{\prime}(s) d s \delta_{0}\right) f_{0}}{\sigma^{2}}
$$

and $\Lambda(r)$ is defined by Equation (7).

Case 2: if $\tau=1 / 2$, then $\widetilde{\gamma}_{n} \Rightarrow \widetilde{\gamma}\left(\gamma_{0}, \delta_{0}\right)$ and $\widetilde{\gamma}\left(\gamma_{0}, \delta_{0}\right)$ is a random variable that maximizes $\widetilde{Q}\left(\gamma, \gamma_{0}, \delta_{0}\right)$ where

$$
\widetilde{Q}\left(\gamma, \gamma_{0}, \delta_{0}\right)=\widetilde{\Gamma}_{1}^{\prime}(\gamma)\left(\widetilde{G}_{22}(\gamma)-\left(\begin{array}{c}
\widetilde{G}_{21}(\gamma) \\
\widetilde{G}_{23}(\gamma)
\end{array}\right)^{\prime}\left(\begin{array}{c}
\widetilde{G}_{11}, \widetilde{G}_{13} \\
\widetilde{G}_{31}, \widetilde{G}_{33}
\end{array}\right)^{-1}\left(\begin{array}{c}
\widetilde{G}_{12}(\gamma) \\
\widetilde{G}_{32}(\gamma)
\end{array}\right)\right)^{-1} \widetilde{\Gamma}_{1}(\gamma)
$$

with

$$
\widetilde{\Gamma}_{1}(\gamma)=\widetilde{\Gamma}(\gamma)+\left(\widetilde{G}_{22}(\gamma)-\left(\begin{array}{c}
\widetilde{G}_{21}(\gamma) \\
\widetilde{G}_{23}(\gamma)
\end{array}\right)^{\prime}\left(\begin{array}{c}
\widetilde{G}_{11}, \widetilde{G}_{13} \\
\widetilde{G}_{31}, \widetilde{G}_{33}
\end{array}\right)^{-1}\left(\begin{array}{c}
\widetilde{G}_{12}(\gamma) \\
\widetilde{G}_{32}(\gamma)
\end{array}\right)\right) \delta_{0}
$$

and

$$
\widetilde{\Gamma}(\gamma)=\widetilde{\phi}_{2}(\gamma)-\left(\begin{array}{c}
\widetilde{G}_{21}(\gamma) \\
\widetilde{G}_{23}(\gamma)
\end{array}\right)^{\prime}\left(\begin{array}{c}
\widetilde{G}_{11}, \widetilde{G}_{13} \\
\widetilde{G}_{31}, \widetilde{G}_{33}
\end{array}\right)^{-1}\left(\begin{array}{c}
\widetilde{\phi}_{1}(\gamma) \\
\widetilde{\phi}_{3}(\gamma)
\end{array}\right)
$$


where the random matrix $\widetilde{G}(\gamma)=\left(\begin{array}{c}\widetilde{G}_{11}(\gamma), \widetilde{G}_{12}(\gamma), \widetilde{G}_{13}(\gamma) \\ \widetilde{G}_{21}(\gamma), \widetilde{G}_{22}(\gamma), \widetilde{G}_{23}(\gamma) \\ \widetilde{G}_{31}(\gamma), \widetilde{G}_{32}(\gamma), \widetilde{G}_{33}(\gamma)\end{array}\right)$ and the random vector $\widetilde{\phi}(\gamma)=\left(\begin{array}{c}\widetilde{\phi}_{1}(\gamma) \\ \widetilde{\phi}_{2}(\gamma) \\ \widetilde{\phi}_{3}(\gamma)\end{array}\right)$ are specified in Lemma B.4 of the appendix.

Theorem 4.2 establishes the convergence results for the FGLS estimator $\widetilde{\gamma}_{n}$. If $-1 / 2<\tau<1 / 2$, the threshold value can be consistently estimated and its limiting distribution depends on the persistence parameter $\rho$. Note that $\widetilde{\lambda}=\left(1+\rho^{2}\right) \lambda$, implying that the FGLS estimator is more efficient than the profiled LS estimator when $\rho \neq 0$. The simulations in Section 5 demonstrate this result.

\subsection{Generalized Sup-Wald Statistics}

Testing for the existence of the threshold effect in a cointegration regression is challenging since it is a joint hypothesis problem (see Balke and Fomby, 1997). For example, when testing for the existence of threshold effects, the statistics based on error correction models (ECM) need to assume the model is a cointegrating regression. Therefore, the rejection of the null hypothesis does not necessarily indicate that there is a threshold effect. It may mean that the regression is a spurious relationship. Thanks to the robustness of the FGLS estimator, we can design a generalized sup-Wald test statistic based on this estimator to test for the existence of threshold effects, without any requirement on the stationarity of the error term.

We consider to test the following hypothesis:

$$
H_{0}: \delta_{n}=0 \quad \text { v.s. } \quad H_{1}: \delta_{n} \neq 0 .
$$

Under the null, after transformation, the model is

$$
\widetilde{y}_{t}=\alpha^{\prime} \widetilde{x}_{t}+\beta^{\prime} \widetilde{z}_{t}+\widetilde{\eta}_{t}
$$

while under the alternative, the transformed model is

$$
\widetilde{y}_{t}=\alpha^{\prime} \widetilde{x}_{t}+\delta_{n}^{\prime} \widetilde{x}_{t}\left(\gamma_{0}\right)+\beta^{\prime} \widetilde{z}_{t}+\widetilde{\eta}_{t}=\widetilde{\theta}^{\prime} \widetilde{A}_{1 t}\left(\gamma_{0}\right)+\widetilde{\eta}_{t}
$$

Let $\widetilde{V_{1}}=(\widetilde{X}, \widetilde{Z})$, then a generalized Wald statistic can be defined as

$$
\widetilde{T}_{n}(\gamma)=\widetilde{\delta}_{n}(\gamma)^{\prime}(\widetilde{X}(\gamma)(I-\widetilde{P}(\gamma)) \widetilde{X}(\gamma)) \widetilde{\delta}_{n}(\gamma) / \widetilde{\sigma}^{2}
$$

where $\widetilde{P}(\gamma)$ is the projection matrix for $\widetilde{V}_{1}$ and $\widetilde{\sigma}^{2}=\widetilde{S S R}_{n}(\gamma) / n$.

The generalized sup-Wald statistic is defined as

$$
\widetilde{T}_{n}=\sup _{\gamma \in[\underline{\gamma}, \bar{\gamma}]} \widetilde{T}_{n}(\gamma)
$$


Theorem 4.3 Under Assumptions (4.1), (4.2), (2.3) and (2.4) and $H_{0}: \delta_{n}=0$, the following limiting result holds:

$$
\begin{aligned}
\widetilde{T}_{n} & \Rightarrow \widetilde{T}=\sup _{\gamma \in[\underline{\gamma}, \bar{\gamma}]} \widetilde{T}(\gamma) \\
& =\sup _{\gamma \in[\underline{\gamma}, \bar{\gamma}]} \frac{1}{\sigma^{2}} \widetilde{\Gamma}^{\prime}(\gamma)\left(\widetilde{G}_{22}(\gamma)-\left(\begin{array}{c}
\widetilde{G}_{21}(\gamma) \\
\widetilde{G}_{23}(\gamma)
\end{array}\right)^{\prime}\left(\begin{array}{c}
\widetilde{G}_{11}, \widetilde{G}_{13} \\
\widetilde{G}_{31}, \widetilde{G}_{33}
\end{array}\right)^{-1}\left(\begin{array}{c}
\widetilde{G}_{12}(\gamma) \\
\widetilde{G}_{32}(\gamma)
\end{array}\right)\right)^{-1} \widetilde{\Gamma}(\gamma),
\end{aligned}
$$

where $\widetilde{\Gamma}(\gamma)$ is defined by Equation (24).

Theorem 4.3 establishes the limiting distribution of the generalized sup-Wald test statistic, which is nonstandard and we generate the critical values using a parametric bootstrap method. We first estimate $\widehat{\alpha}$ and $\widehat{\beta}$ using Equation (25) under the null. Then, we obtain the residual terms $\left\{\widehat{\widetilde{\eta}}_{t}\left(\widetilde{\gamma}_{n}\right)\right\}_{t=2}^{n}$ using Equation (26) under

the alternative. We draw a random variable $\widetilde{\eta}_{t}^{b}$ from the sample $\left\{\widehat{\widetilde{\eta}}_{t}\left(\widetilde{\gamma}_{n}\right)\right\}_{t=2}^{n}$ for all $t=2, \ldots, n$, and generate a new sequence $\left\{\widetilde{y}_{t}^{b}\right\}_{t=1}^{n}$ by $\widetilde{y}_{t}^{b}=\widehat{\alpha}^{\prime} \widetilde{x}_{t}+\widehat{\beta}^{\prime} \widetilde{z}_{t}+\widetilde{\eta}_{t}^{b}$. Define $y_{1}^{b}=y_{1}$ and $y_{t}^{b}=\widetilde{y}_{t}^{b}+\widehat{\rho} y_{t-1}^{b}$ for all $t=2, \ldots, n$. Let $\widetilde{T}_{n}^{b}$ be the sup-Wald test calculated from the new data set $\left\{y_{t}^{b}, x_{t}, z_{t}, q_{t}\right\}_{t=2}^{n}$. Under the null, the distribution of $\widetilde{T}_{n}^{b}$ approximates the distribution of $\widetilde{T}_{n}$. The bootstrap $p$-value is obtained by calculating the frequency of simulated $\widetilde{T}_{n}^{b}$ that exceeds $\widetilde{T}_{n}$ when the number of simulations is sufficiently large. Following Hansen (1996), one can show that the generated $p$-value converges to the true size.

One can use the generalized sup-Wald statistic $\widetilde{T}_{n}$ to construct robust CIs for the threshold estimators following the procedures described in Section 3.2.

\section{Simulations}

This section demonstrates the finite sample performance of the estimators and test statistics through two simulation experiments.

Experiment 1: In this experiment, we examine the finite sample performance of the profiled LS estimator and the FGLS estimator under different model settings. The data generating process is given by

$$
y_{t}=\alpha x_{t}+\delta_{n} x_{t}\left(q_{t} \leq \gamma_{0}\right)+\beta v_{t}+u_{t}
$$

where $x_{t}=x_{t-1}+v_{t}$ and $u_{t}=\rho u_{t-1}+\varepsilon_{t}$. The threshold variable $q_{t}$ is generated by an $A R(1)$ process: $q_{t}=0.5 q_{t-1}+e_{t}$. The innovation processes $v_{t}, \varepsilon_{t}$ and $e_{t}$ follow i.i.d. $N(0,1)$ and are independent of each other. The true threshold value is set as $\gamma_{0}=0$ and the coefficient $\alpha$ is set as 1 . The parameter $\beta$ is set as 0 or 0.5 , which controls the correlation between $x_{t}$ and $u_{t}$. To check the impact of the serial correlation of $u_{t}$ on the estimation, we set $\rho$ as either $0,1,0.95$ or -0.95 . Moreover, we set the size of the threshold effect as $\delta_{n}=2 n^{-1 / 2-\tau}$ with $\tau$ chosen as either 0 or 0.5 , corresponding to the case with identification or the case with weak identification. The number of replications is $N=1000$. The sample size $n$ is set as 100,200 or 400 . We let the number of lags $K=5$ 
when construct the FGLS estimator. ${ }^{8}$

Table 1 reports the mean square errors (MSE) of the profiled LS estimator and the FGLS estimator for the threshold value.

\section{Here insert Table 1}

Specifically, Panel A of Table 1 displays the results when $\tau=0$, i.e., the case with identification. From this panel, one can find that the MSE of the profiled LS estimator $\widehat{\gamma}$ decreases to zero as sample size increases when $\rho<1$, but it does not when $\rho=1$. On the other hand, the MSE of the FGLS estimator $\widetilde{\gamma}$ decays to zero regardless of whether $\rho<1$ or $\rho=1$. Of particular interest is that the FGLS estimator performs better than the profiled LS estimator when serial correlation and regressor endogeneity exist. Panel B reports the results when $\tau=0.5$, i.e., the case with weak identification. From this panel, one can find that neither the MSE of profiled LS estimator or that of FGLS estimator converges to zero. However, the FGLS estimator has smaller MSE than the profiled LS estimator when serial correlation and regressor endogeneity exist. In summary, we conclude that the FGLS estimator does improve the estimation efficiency.

Experiment 2: Through this experiment, we examine the performance of the test statistics and the model selection procedure. The data generating process is the same as in Simulation 1, but we consider the case of $\delta_{n}=0$ to evaluate the size performance of the test statistics.

Table 2 reports the size performance of the sup-Wald statistic $T_{n}$ and the generalized sup-Wald statistic $\widetilde{T}_{n}$, which are constructed using the profiled LS estimator and the FGLS estimator, respectively. Comparing Panel A with Panel B, one can see that $\widetilde{T}_{n}$ has better size performance than $T_{n}$ when serial correlation or regressor endogeneity exists. In an unreported result with the sample size $n=1000$, the rejection rates of $\widetilde{T}_{n}$ are close to the size, but not for $T_{n}$.

\section{Here insert Table 2}

Tables 3 and 4 report the power performance for $T_{n}$ and $\widetilde{T}_{n}$, respectively. In both tables, Panel A shows the results for the case with identification and Panel B shows the results for the case with weak identification.

\section{Here insert Tables 3 and 4}

When the threshold effect is identified, both $T_{n}$ and $\widetilde{T}_{n}$ seem to be consistent since their power converges to one very quickly as the sample size increases. However, $\widetilde{T}_{n}$ performs better than $T_{n}$ when there exists serial correlation or model endogeneity. Thus, we recommend to use the generalized sup-Wald statistic $\widetilde{T}_{n}$ for testing the existence of threshold effects in practice.

When the threshold effect is only weakly identified, both statistics seem to have low power even when the sample size is 400, which is consistent to the results of Theorem 3.2. In such case, we recommend to use the model selection procedure described in Section 3.2 to detect the weak identification at first. In our simulation, 
we choose $(\ln (n))^{2}$ as the tuning parameter $\kappa_{n}$ and judge that the model is weakly identified if the test statistic is smaller than $\kappa_{n}$. From the columns corresponding to $(\ln (n))^{2}$ in Tables 3 and 4 , one can find that the model selection procedure based on $\widetilde{T}_{n}$ works very well.

\section{Conclusion}

In the literature, statistical theory for threshold models with stationary explanatory variables has been well developed by Hansen $(1996,2000)$. However, in empirical macroeconomics and finance, many explanatory variables are nonstationary. This paper establishes the asymptotic theory for threshold models with nonstationary regressors under the diminishing threshold effect assumption. What is more important, we provide a method to construct robust confidence intervals for the threshold estimators, which have the correct asymptotic size irrespective of the magnitude of the threshold effect. The study can also be related to the literature of nonlinear cointegration as the model allows for a threshold effect in the cointegration relationship.

There are several directions open for further work. First, it may be interesting to develop a more general model with multiple threshold effects, each with a different identification strength. A sequential procedure can be applied to determine the number of regimes and their identification strengths. Second, the model can be extended to allow for stationary regressors. Moreover, there are many interesting applications. For example, one can use the model to study the regime-sensitive Taylor rule, where the dependent variable $y_{t}$ could be the short-term interest rate, the explanatory variables $x_{t}$ could be macroeconomic variables such as the inflation rate and the unemployment rate, and the threshold variable $q_{t}$ could be the GDP growth rate. Another application is to model the regime-dependent predictability of the fundamental ratios, such as the dividend-price ratio and the earning-price ratio, to equity returns. The threshold variable could be a variable indicating the status of the economy. All these are left for future studies.

\section{NOTES}

1. The model could be extended to include an intercept term and stationary regressors.

2. Loosely speaking, if the response variable $y_{t}$ is generated by a nonlinear transformation of integrated regressors $x_{t}$ plus a stationary error term, then there exists a nonlinear cointegrating relationship between $y_{t}$ and $x_{t}$.

3. Marmer (2008) shows that linear regression techniques actually may lead to spurious forecasts if there exist small departures from the linear structure, but improvements of the forecast accuracy are possible if the nonlinear components are properly considered.

4. See Elliott and Müller (2007) for weak identification in break-point models; Cheng (2008) and Shi and Phillips (2012) for weak identification in general nonlinear models.

5. The assumption (2.2) can be relaxed under certain circumstances. For instance, $u_{t}$ could be generalized to 
follow a linear moving average process of finite order $l$. However, fully generalizing the model to allow for serially correlated errors would involve substantial added complexity (see Gonzalo and Pitarakis, 2006).

6. In an early version of this paper, we have also established the limiting distribution of the coefficient estimators $\widehat{\theta}\left(\widehat{\gamma}_{n}\right)$. We show that, if $-1 / 2<\tau<1 / 2$, the limiting distribution of the coefficients estimators is mixed normal, which makes conventional $t$-test and chi-square tests applicable, whereas if $\tau=1 / 2$, the limiting result contains a bias term as a result of the inconsistency of $\widehat{\gamma}_{n}$.

7. Structural spurious regressions can be due to integrated measurement errors and missing integrated regressors. See Choi, Hu and Ogaki (2008).

8. Other values for $K$, such as 10 and 15 , are also applied with little change in the results.

\section{REFERENCES}

Andrews, D.W.K. \& C. J. McDermott (1995) Nonlinear econometric models with deterministically trending variables. Review of Economic Studies, 62(3), 343-60.

Balke, N. \& T. Fomby (1997) Threshold cointegration. International Economics Review, 8, 627-645.

Bierens H. \& L. Martins (2010) Time-varying cointegration. Econometric Theory, 26, 1453-1490.

Cai, Z., Q. Li \& J.Y. Park (2009) Functional-coefficient models for nonstationary time series data. Journal of Econometrics, 148, 101-113.

Campbell, J.Y. \& M. Yogo (2006) Efficient tests of stock return predictability. Journal of Financial Economics, $81,27-60$.

Caner, M. \& B.E. Hansen (2001) Threshold autoregression with a unit root. Econometrica, 69, 1555-1596.

Chen, H. (2013) Robust Estimation and Inference for Threshold Models with Integrated Regressors. Working Papers, Xiamen University. Available at SSRN: http://ssrn.com/abstract=2287442.

Cheng, X. (2008) Robust confidence intervals in nonlinear regression under weak identification. Manuscript, Department of Economics, Yale University.

Chan, K.S. (1993) Consistency and limiting distribution of the least squares estimator of a threshold autoregressive Model. Annals of Statistics, 21, 520-533.

Choi, C.Y., L. Hu \& M. Ogaki (2008) Robust estimation for structural spurious regressions and a Hausman-type cointegration test. Journal of Econometrics, 142, 327-351.

Choi, In \& P. Saikkonen (2010) Test of nonlinear cointegration. Econometric Theory, 26, 682-709. 
Durlauf, S.N. \& P.A. Johnson (1995) Multiple regimes and cross-country growth behavior. Journal of Applied Econometrics, 10, 365-84.

Elliott, G. \& U.K. Müller (2007) Confidence sets for the date of a single break in linear time series regressions. Journal of Econometrics, 141(2), 1196-1218.

Gao, J., M. L. King, Z. Lu \& D. Tjøstheim (2009a) Specification testing in nonstationary time series autoregression. Annals of Statistics, 37, 3893-3928.

Gao, J., M. L. King, Z. Lu \& D. Tjøstheim (2009b) Nonparametric specification testing for nonlinear time series with nonstationarity. Econometric Theory, 25, 1869-1892.

Gonzalo, J. \& J. Pitarakis (2002) Estimation and model selection based inference in single and multiple threshold models. Journal of Econometrics, 110, 319-352.

Gonzalo, J. \& J.Y. Pitarakis (2006) Threshold effects in cointegrating regressions. Oxford Bulletin of Economics and Statistics, 813-833.

Gonzalo, J. \& J.Y. Pitarakis (2012) Regime specific predictability in predictive regressions. Journal of Business and Economic Statistics, 30(2), 229-241.

Hansen, B.E. (1996) Inference when a nuisance parameter is not identified under the null hypothesis. Econometrica, 64, 413-430.

Hansen, B.E. (1997) Inference in TAR models. Studies in Nonlinear Dynamics and Econometrics, 2, 1-14.

Hansen, B.E. (2000) Sample splitting and threshold estimation. Econometrica, 68, 575-603.

Li, D. \& S. Q. Ling (2012) On the least squares estimation of multiple-regime threshold autoregressive models. Journal of Econometrics, 167, 240-253.

Marmer, V. (2008) Nonlinearity, nonstationarity, and spurious forecasts, Journal of Econometrics, 142(1), 1-27.

Park, J.Y. (1992) Canonical cointegrating regressions. Econometrica, 60, 119-143.

Park, J.Y. \& S. Hahn (1999) Cointegration regression with time varying coefficients. Econometric Theory, 15, 664-703.

Park, J.Y. \& P.C.B. Phillips (2001) Nonlinear regressions with integrated time series. Econometrica, 69(1), $117-161$.

Perron, P. \& T. Yabu (2009) Testing for shifts in trend with an integrated or stationary noise component. Journal of Business and Economics Statistics, 27(3), 369-396.

Phillips, P.C.B. \& S.N. Durlauf (1986) Multiple time series regression with integrated processes. Review of Economic Studies, 53, 474-95. 
Phillips, P.C.B. \& J.Y. Park (1988) Asymptotic equivalence of ordinary least squares and generalized Least squares in regressions with integrated regressors. Journal of the American Statistical Association, 83, $111-115$.

Phillips, P.C.B. \& B.E. Hansen (1990) Statistical inference in instrumental variables regression with I(1) processes. Review of Economic Studies, 57, 99-125.

Phillips, P.C.B. \& D.J. Hodgson (1994) Spurious regression and generalized least squares. Econometric Theory, 10, 957-958.

Phillips, P.C.B. \& J.H Lee (2013) Predictive regression under varying degrees of persistence and robust longhorizon regression. Journal of Econometrics, in press.

Potter, S.M. (1995) A nonlinear approach to US GNP. Journal of Applied Econometrics, 2, 109-25.

Saikkonen, P. (1991) Asymptotically efficient estimation of cointegration regressions. Econometric Theory, 7, $1-20$.

Seo, M. \& O. Linton (2007) A smoothed least squares estimator for threshold regression models. Journal of Econometrics, 141, 704-735.

Shao, Q. \& C. Lu (1987) Strong approximation for partial sums of weakly dependent random variables. Scientia Sinica, 15, 576-587.

Shi, X. \& P.C.B. Phillips (2012) Nonlinear cointegration regression under weak identification. Econometric Theory, 28(1), 1-39.

Wang, Q. \& P.C.B. Phillips (2009) Structural nonparametric cointegrating regression. Econometrica, 77(6), 1901-1948.

Xiao, Z. (2009) Functional-coefficient cointegration models. Journal of Econometrics, 152(2), 81-92.

Yu, P. (2012) Likelihood estimation and inference in threshold regression. Journal of Econometrics, 167, 274294.

\section{Appendix: Mathematical Proofs}

In this appendix, Part A provides the proofs related to the basic model while Part B is for the generalized model. To save space, we skip the details for some intermediary results and a more detailed presentation can be found in Chen (2013).

\section{Part A}


LEMMA A.1. Under Assumptions $(2.1)-(2.3)$, for any $\gamma \in[\underline{\gamma}, \bar{\gamma}]$, we have $\frac{1}{\sqrt{n}} \sum_{t=1}^{[n s]} I_{t}\left(q_{t} \leq \gamma\right) u_{t} \Rightarrow$ $\sigma_{u} W(s, F(\gamma))$.

LEMMA A.2. Under Assumptions (2.1) - (2.4), for any $\gamma \in[\underline{\gamma}, \bar{\gamma}]$, we have

$$
\frac{1}{n} \sum_{t=1}^{n} x_{t} I_{t}(\gamma) u_{t} \Rightarrow \sigma_{u} \int \mathbf{B}_{v}(s) d W(s, F(\gamma))
$$

LEMMA A.3. Under Assumptions (2.1) - (2.4), for any $\gamma \in[\underline{\gamma}, \bar{\gamma}]$, as $n \rightarrow \infty$, we have

(i) $n^{-2} \sum_{t=1}^{n} A_{t}(\gamma) A_{t}^{\prime}(\gamma) \stackrel{p}{\rightarrow} M(\gamma)$,

(ii) $n^{-1} \sum_{t=1}^{n} A_{t}(\gamma) u_{t} \Rightarrow \sigma_{u}\left(\begin{array}{c}\int \mathbf{B}_{v}(s) d W(s) \\ \int \mathbf{B}_{v}(s) d W(s, F(\gamma))\end{array}\right)$,

where $A_{t}(\gamma)$ is defined in Equation (5) and $M(\gamma)=\left(\begin{array}{cc}\int \mathbf{B}_{v}(s) \mathbf{B}_{v}^{\prime}(s) d s & F(\gamma) \int \mathbf{B}_{v}(s) \mathbf{B}_{v}^{\prime}(s) d s \\ F(\gamma) \int \mathbf{B}_{v}(s) \mathbf{B}_{v}^{\prime}(s) d s & F(\gamma) \int \mathbf{B}_{v}(s) \mathbf{B}_{v}^{\prime}(s) d s\end{array}\right)$.

LEMMA A.4. Under Assumptions (2.1) - (2.4), we have

$$
n\left(\widehat{\theta}\left(\gamma_{0}\right)-\theta\right) \Rightarrow \sigma_{u} M\left(\gamma_{0}\right)^{-1}\left(\begin{array}{c}
\int \mathbf{B}_{v}(s) d W(s) \\
\int \mathbf{B}_{v}(s) d W\left(s, F\left(\gamma_{0}\right)\right)
\end{array}\right)
$$

For any $\gamma \neq \gamma_{0}$, if $-1 / 2<\tau<1 / 2$, we have

$$
n^{\tau+1 / 2}(\widehat{\theta}(\gamma)-\theta) \Rightarrow M(\gamma)^{-1} \Pi\left(\gamma, \gamma_{0}, \delta_{0}\right)
$$

if $\tau=1 / 2$,

$$
n(\widehat{\theta}(\gamma)-\theta) \Rightarrow \sigma_{u} M(\gamma)^{-1}\left(\begin{array}{c}
\int \mathbf{B}_{v}(s) d W(s) \\
\int \mathbf{B}_{v}(s) d W(s, F(\gamma))
\end{array}\right)+M(\gamma)^{-1} \Pi\left(\gamma, \gamma_{0}, \delta_{0}\right)
$$

where $\Pi\left(\gamma, \gamma_{0}, \delta_{0}\right)=-\left(\begin{array}{c}\left(F(\gamma)-F\left(\gamma_{0}\right)\right) \int \mathbf{B}_{v}(s) \mathbf{B}_{v}^{\prime}(s) d s \\ \left(F(\gamma)-F\left(\gamma_{0} \wedge \gamma\right)\right) \int \mathbf{B}_{v}(s) \mathbf{B}_{v}^{\prime}(s) d s\end{array}\right) \delta_{0}$.

Proof of Lemmas A.1-A.4: The proofs are standard and are skipped here.

LEMMA A.5. If $-1 / 2<\tau<1 / 2$, we have $\widehat{\gamma}_{n} \stackrel{p}{\rightarrow} \gamma_{0}$.

Proof: To establish the consistency of $\widehat{\gamma}_{n}$, we need to prove $\operatorname{Pr}\left(\left|\widehat{\gamma}_{n}-\gamma_{0}\right|>\varepsilon\right) \rightarrow 0$ for any $\varepsilon>0$. Denote $B(\epsilon)=\left\{\gamma:\left|\gamma-\gamma_{0}\right|>\varepsilon\right\}$ and $\bar{B}(\epsilon)=[\underline{\gamma}, \bar{\gamma}] \backslash B(\epsilon)$. By the definition of $\widehat{\gamma}_{n}$, we have

$$
\begin{aligned}
\operatorname{Pr}\left(\left|\widehat{\gamma}_{n}-\gamma_{0}\right|\right. & >\varepsilon)=\operatorname{Pr}\left(\inf _{\gamma \in B(\epsilon)} S S R_{n}(\gamma)<\inf _{\gamma \in \bar{B}(\epsilon)} S S R_{n}(\gamma)\right) \leq \operatorname{Pr}\left(\inf _{\gamma \in B(\epsilon)} S S R_{n}(\gamma)<S S R_{n}\left(\gamma_{0}\right)\right) \\
& =\operatorname{Pr}\left(\inf _{\gamma \in B(\epsilon)} n^{2 \tau-1}\left(S S R_{n}(\gamma)-S S R_{n}\left(\gamma_{0}\right)\right)<0\right) .
\end{aligned}
$$

Thus, to prove $\operatorname{Pr}\left(\left|\widetilde{\gamma}_{n}-\gamma_{0}\right|>\varepsilon\right) \rightarrow 0$, it suffices to show that $\inf _{\gamma \in B(\epsilon)} n^{2 \tau-1}\left(S S R_{n}(\gamma)-S S R_{n}\left(\gamma_{0}\right)\right)>0$ with 
probability 1.

Rewrite Equation (2) as a matrix compacted form: $Y=X^{\prime} \alpha+X\left(\gamma_{0}\right)^{\prime} \delta_{n}+u$, where $Y, X, X\left(\gamma_{0}\right)$ and $u$ stack $y_{t}, x_{t}, x_{t} I\left(q_{t} \leq \gamma_{0}\right)$ and $u_{t}$, respectively. Denote $X^{*}(\gamma)=(X(\gamma), X-X(\gamma))$ and define its projection matrix $P_{\gamma}^{*}=X^{*}(\gamma)\left(X^{*}(\gamma)^{\prime} X^{*}(\gamma)\right)^{-1} X^{*}(\gamma)^{\prime}$. By some simple algebra, we have

$$
S S R_{n}(\gamma)=Y^{\prime}\left(I-P_{\gamma}^{*}\right) Y=\delta_{n}^{\prime} X\left(\gamma_{0}\right)^{\prime}\left(I-P_{\gamma}^{*}\right) X\left(\gamma_{0}\right) \delta_{n}+2 \delta_{n}^{\prime} X\left(\gamma_{0}\right)^{\prime}\left(I-P_{\gamma}^{*}\right) u+u^{\prime}\left(I-P_{\gamma}^{*}\right) u
$$

where the second equation uses the fact that $X^{\prime}\left(I-P_{\gamma}^{*}\right)=0$, since $X$ is a linear combination of $(X(\gamma), X-X(\gamma))$.

When $\gamma=\gamma_{0}$, we have $S S R_{n}\left(\gamma_{0}\right)=u^{\prime}\left(I-P_{\gamma_{0}}^{*}\right) u$, and it follows that

$$
\begin{aligned}
n^{2 \tau-1}\left(S S R_{n}(\gamma)-S S R_{n}\left(\gamma_{0}\right)\right)= & n^{-1+2 \tau} \delta_{n}^{\prime} X\left(\gamma_{0}\right)^{\prime}\left(I-P_{\gamma}^{*}\right) X\left(\gamma_{0}\right) \delta_{n}+n^{-1+2 \tau} 2 \delta_{n}^{\prime} X\left(\gamma_{0}\right)^{\prime}\left(I-P_{\gamma}^{*}\right) u \\
& +n^{-1+2 \tau}\left(u^{\prime}\left(I-P_{\gamma}^{*}\right) u-u^{\prime}\left(I-P_{\gamma_{0}}^{*}\right) u\right) \equiv S_{1}+S_{2}+S_{3}, \text { say. }
\end{aligned}
$$

Next, we will show that $S_{1}+S_{2}+S_{3}$ uniformly converges to a function $b(\gamma)$ which is positive for any $\gamma \in B(\epsilon)$. Given $-1 / 2<\tau<1 / 2$, by Lemma A.3, it can be shown that

$$
\begin{aligned}
& S_{2}=n^{-1 / 2+\tau} 2\left(n^{1 / 2+\tau} \delta_{n}\right)^{\prime} \frac{1}{n} X\left(\gamma_{0}\right)^{\prime}\left(I-P_{\gamma}^{*}\right) u=n^{-1 / 2+\tau} 2 \delta_{0}^{\prime} \frac{1}{n} X\left(\gamma_{0}\right)^{\prime}\left(I-P_{\gamma}^{*}\right) u=O_{p}\left(n^{-1 / 2+\tau}\right) \stackrel{p}{\rightarrow} 0, \\
& S_{3}=n^{-1+2 \tau}\left(u^{\prime}\left(I-P_{\gamma}^{*}\right) u-u^{\prime}\left(I-P_{\gamma_{0}}^{*}\right) u\right)=n^{-1+2 \tau}\left(u^{\prime} P_{\gamma_{0}}^{*} u-u^{\prime} P_{\gamma}^{*} u\right)=O_{p}\left(n^{-1+2 \tau}\right) \stackrel{p}{\rightarrow} 0 .
\end{aligned}
$$

Using a similar argument of Lemma A.5 in Hansen(2000), we can show, for any $\gamma \geq \gamma_{0}$,

$$
S_{1}=n^{-1+2 \tau} \delta_{n}^{\prime} X^{\prime}\left(\gamma_{0}\right)\left(I-P_{\gamma}^{*}\right) X\left(\gamma_{0}\right) \delta_{n} \stackrel{p}{\rightarrow}\left(F\left(\gamma_{0}\right)-F\left(\gamma_{0}\right) F(\gamma)^{-1} F\left(\gamma_{0}\right)\right) \delta_{0}^{\prime}\left(\int \mathbf{B}_{v}(s) \mathbf{B}_{v}^{\prime}(s) d s\right) \delta_{0} \equiv b_{1}(\gamma)
$$

uniformly. Since $\left(F\left(\gamma_{0}\right)-F\left(\gamma_{0}\right) F(\gamma)^{-1} F\left(\gamma_{0}\right)\right) \geq 0$ and $\int \mathbf{B}_{v}(s) \mathbf{B}_{v}^{\prime}(s) d s$ is positive definite random matrix, $b_{1}(\gamma) \geq 0$ and the equality holds if and only if $\gamma=\gamma_{0}$.

For $\gamma \leq \gamma_{0}$, we can show $S_{1} \stackrel{p}{\rightarrow}\left(F\left(\gamma_{0}\right)-F(\gamma)\right) \delta_{0}^{\prime}\left(\int \mathbf{B}_{v}(s) \mathbf{B}_{v}^{\prime}(s) d s\right) \delta_{0} \equiv b_{2}(\gamma)$ uniformly, where $b_{2}(\gamma) \geq 0$ and the equality holds if and only if $\gamma=\gamma_{0}$. Define $b(\gamma)=b_{1}(\gamma) I\left(\gamma \geq \gamma_{0}\right)+b_{2}(\gamma) I\left(\gamma \leq \gamma_{0}\right)$, and we have shown that $n^{-1+2 \tau}\left(S S R_{n}(\gamma)-S S R_{n}\left(\gamma_{0}\right)\right) \stackrel{p}{\rightarrow} b(\gamma)$ uniformly for any $\gamma \in[\underline{\gamma}, \bar{\gamma}]$ and $b(\gamma)$ is strictly positive when $\gamma \in B(\epsilon)$. Thus, $\operatorname{Pr}\left(\inf _{\gamma \in B(\epsilon)} n^{2 \tau-1}\left(S S R_{n}(\gamma)-S S R_{n}\left(\gamma_{0}\right)\right)>0\right) \rightarrow 1$.

Proof of Theorem 3.1: We first prove the limiting results for the case with $-1 / 2<\tau<1 / 2$. Let $a_{n}=n^{1-2 \tau}$. To prove $\widehat{\gamma}_{n}$ converge to $\gamma_{0}$ with rate $a_{n}$, we need to prove that $a_{n}\left|\widehat{\gamma}_{n}-\gamma_{0}\right|=O_{p}(1)$, or there exists a constant $\bar{v}>0, \lim _{n \rightarrow \infty} \operatorname{Pr}\left(\left|\widehat{\gamma}_{n}-\gamma_{0}\right| \leq \bar{v} / a_{n}\right)=1$. For any $B>0$, define $V_{B}=\left\{\gamma:\left|\gamma-\gamma_{0}\right|<B\right\}$. When $n$ is large enough, we have $\bar{v} / a_{n}<B$. Since $\widehat{\gamma}_{n} \stackrel{p}{\rightarrow} \gamma_{0}$ according to Lemma A.5, $\operatorname{Pr}\left(\left\{\widehat{\gamma}_{n} \in V_{B}\right\}\right) \stackrel{p}{\rightarrow} 1$. Therefore, we only need to examine the limiting behavior of $\gamma$ in $V_{B}$.

Define a subset $V_{B}(\bar{v})=\left\{\gamma: \bar{v} / a_{n}<\left|\gamma-\gamma_{0}\right|<B\right\}$. Thus, $V_{B}(\bar{v}) \subset V_{B}$. To prove $\operatorname{Pr}\left(\left|\widehat{\gamma}_{n}-\gamma_{0}\right| \leq \bar{v} / a_{n}\right)=$ 1 , we just need to prove $\operatorname{Pr}\left(\widehat{\gamma}_{n} \in V_{B}(\bar{v})\right)=0$. Let $\widehat{\theta}$ and $\widehat{\delta}$ be the estimation of $\widehat{\theta}\left(\widehat{\gamma}_{n}\right)$ and $\widehat{\delta}\left(\widehat{\gamma}_{n}\right)$. Also, we 
denote $S S R_{n}^{*}(\gamma)=\sum_{t=1}^{n}\left(y_{t}-\widehat{\theta}^{\prime} A_{t}(\gamma)\right)^{2}$ and $S S R_{n}^{*}\left(\gamma_{0}\right)=\sum_{t=1}^{n}\left(y_{t}-\widehat{\theta}^{\prime} A_{t}\left(\gamma_{0}\right)\right)^{2}$. By the definition of $\widehat{\gamma}_{n}$, we have $S S R_{n}^{*}\left(\widehat{\gamma}_{n}\right) \leq S S R_{n}^{*}\left(\gamma_{0}\right)$. Hence, it suffices to prove that for any $\gamma \in V_{B}(\bar{v}), S S R_{n}^{*}(\gamma)>S S R_{n}^{*}\left(\gamma^{0}\right)$ with probability 1 .

We consider the case of $\gamma>\gamma^{0}$ first. Using an argument of symmetry, we can, without loss of generality, prove the result for the case of $\gamma<\gamma_{0}$. Note that

$$
\begin{aligned}
& S S R_{n}^{*}(\gamma)-S S R_{n}^{*}\left(\gamma_{0}\right)=\sum_{t=1}^{n}\left(y_{t}-\widehat{\theta}^{\prime} A_{t}(\gamma)\right)^{2}-\sum_{t=1}^{n}\left(y_{t}-\widehat{\theta}^{\prime} A_{t}\left(\gamma_{0}\right)\right)^{2} \\
= & \sum_{t=1}^{n} \delta_{n}^{\prime}\left(x_{t}(\gamma)-x_{t}\left(\gamma_{0}\right)\right)\left(x_{t}(\gamma)-x_{t}\left(\gamma_{0}\right)\right)^{\prime} \delta_{n}-2 \delta_{n}^{\prime} \sum_{t=1}^{n}\left(x_{t}(\gamma)-x_{t}\left(\gamma_{0}\right)\right) u \\
& +2 \widehat{\delta}^{\prime} \sum_{t=1}^{n}\left(x_{t}(\gamma)-x_{t}\left(\gamma_{0}\right)\right)\left(x_{t}(\gamma)-x_{t}\left(\gamma_{0}\right)\right)^{\prime}(\widehat{\theta}-\theta) \\
& +2 \sum_{t=1}^{n}\left(\widehat{\delta}+\delta_{n}\right)^{\prime}\left(x_{t}(\gamma)-x_{t}\left(\gamma_{0}\right)\right)\left(x_{t}(\gamma)-x_{t}\left(\gamma_{0}\right)\right)^{\prime}\left(\widehat{\delta}-\delta_{n}\right)+2\left(\delta_{n}^{\prime}-\widehat{\delta}^{\prime}\right) \sum_{t=1}^{n}\left(x_{t}(\gamma)-x_{t}\left(\gamma_{0}\right)\right) u \\
\equiv & R_{1}-R_{2}+R_{3}+R_{4}+R_{5}, \text { say. }
\end{aligned}
$$

Next, we show that $\frac{R_{1}+R_{2}+R_{3}+R_{4}+R_{5}}{a_{n}\left(\gamma-\gamma_{0}\right)}$ converges to a positive random variable almost surely by studying the limiting behavior for each term specifically. First, note that

$$
\begin{aligned}
\frac{R_{1}}{a_{n}} & =\frac{1}{a_{n}} \sum_{t=1}^{n} \delta_{n}^{\prime}\left(x_{t}(\gamma)-x_{t}\left(\gamma_{0}\right)\right)\left(x_{t}(\gamma)-x_{t}\left(\gamma_{0}\right)\right)^{\prime} \delta_{n}=\left(F(\gamma)-F\left(\gamma_{0}\right)\right) \delta_{0}^{\prime} \int \mathbf{B}_{v}(s) \mathbf{B}_{v}^{\prime}(s) d s \delta_{0}+o_{p}(1) \\
& =f\left(\gamma_{0}\right)\left(\gamma-\gamma_{0}\right) \delta_{0}^{\prime}\left(\int \mathbf{B}_{v}(s) \mathbf{B}_{v}^{\prime}(s) d s\right) \delta_{0}+o_{p}(1) .
\end{aligned}
$$

The last equation uses the first-order Taylor approximation of $F(\gamma)$ around $\gamma_{0}$.

Noting that $\bar{v} / a_{n}<\left|\gamma-\gamma_{0}\right|<B$ and $a_{n}=n^{1-2 \tau}$ with $\tau<1 / 2$, we have $\sqrt{\bar{v}}<\sqrt{a_{n}} \sqrt{\left(\left|\gamma-\gamma_{0}\right|\right)}$. Thus, there exists $k>0$, such that

$$
\frac{R_{2}}{a_{n}\left(\gamma-\gamma_{0}\right)}=\frac{2 \delta_{0}^{\prime} \frac{1}{n} \sum_{t=1}^{n}\left(x_{t}(\gamma)-x_{t}\left(\gamma_{0}\right)\right) u}{\sqrt{a_{n}}\left(\gamma-\gamma_{0}\right)}=O_{p}\left(\frac{1}{\sqrt{a_{n}} \sqrt{\left(\left|\gamma-\gamma_{0}\right|\right)}}\right) \leq k / \sqrt{\bar{v}}
$$

For any $B \rightarrow 0_{+}$, there exists $\bar{v}>0$ and $N$, such that $k / \sqrt{\bar{v}}<f\left(\gamma_{0}\right) \delta_{0}^{\prime} \int \mathbf{B}_{v}(s) \mathbf{B}_{v}^{\prime}(s) d s \delta_{0}$ with probability 1 and $\bar{v} / a_{n}<B$ when $n>N$. Therefore, for any $\gamma \in V_{B}(\bar{v})$, we have

$$
\frac{R_{1}}{a_{n}\left(\gamma-\gamma_{0}\right)}-\frac{R_{2}}{a_{n}\left(\gamma-\gamma_{0}\right)}>0
$$

Furthermore, from Lemma A.4, we have $n^{\tau+1 / 2}\left((\widehat{\theta}-\theta)=O_{p}\left(\widehat{\gamma}_{n}-\gamma_{0}\right)\right.$ and $n^{\tau+1 / 2}\left(\widehat{\delta}_{n}-\delta_{n}\right)=O_{p}\left(\widehat{\gamma}_{n}-\gamma_{0}\right)$, thus

$$
\frac{R_{3}}{a_{n}\left(\gamma-\gamma_{0}\right)}=\frac{2 n^{\tau+1 / 2} \widehat{\delta}_{n}^{\prime} n^{-2} \sum_{t=1}^{n}\left(x_{t}(\gamma)-x_{t}\left(\gamma_{0}\right)\right)\left(x_{t}(\gamma)-x_{t}\left(\gamma_{0}\right)\right)^{\prime} n^{\tau+1 / 2}(\widehat{\theta}-\theta)}{\left(\gamma-\gamma_{0}\right)}=O_{p}\left(\widehat{\gamma}_{n}-\gamma_{0}\right)=o_{p}(1)
$$




$$
\begin{aligned}
\frac{R_{4}}{a_{n}\left(\gamma-\gamma_{0}\right)} & =\frac{2 n^{\tau+1 / 2}\left(\widehat{\delta}+\delta_{n}\right)^{\prime} n^{-2} \sum_{t=1}^{n}\left(x_{t}(\gamma)-x_{t}\left(\gamma_{0}\right)\right)\left(x_{t}(\gamma)-x_{t}\left(\gamma_{0}\right)\right)^{\prime} n^{\tau+1 / 2}\left(\widehat{\delta}-\delta_{n}\right)}{\left(\gamma-\gamma_{0}\right)} \\
& =O_{p}\left(n^{\tau+1 / 2}\left(\widehat{\delta}-\delta_{n}\right)=O_{p}\left(\widehat{\gamma}_{n}-\gamma_{0}\right)=o_{p}(1),\right. \\
\frac{R_{5}}{a_{n}\left(\gamma-\gamma_{0}\right)}= & \frac{2 n^{2 \tau}\left(\delta_{n}^{\prime}-\widehat{\delta}^{\prime}\right) n^{-1} \sum_{t=1}^{n}\left(x_{t}(\gamma)-x_{t}\left(\gamma_{0}\right)\right) u}{\left(\gamma-\gamma_{0}\right)}=O_{p}\left(n^{\tau+1 / 2}\left(\widehat{\delta}-\delta_{n}\right) n^{\tau-1 / 2}\right)=o_{p}(1) .
\end{aligned}
$$

Combining the results of Equations (A.3) through (A.6), we have $\frac{S S R_{n}^{*}(\gamma)-S S R_{n}^{*}\left(\gamma_{0}\right)}{a_{n}\left(\gamma-\gamma_{0}\right)}>0$ with probability 1 for any $\gamma \in V_{B}(\bar{v})$ and $\gamma>\gamma^{0}$. Similarly, we can prove $S S R_{n}^{*}(\gamma)>S S R_{n}^{*}\left(\gamma^{0}\right)$ when $\gamma<\gamma^{0}$ and $\gamma \in V_{B}(\bar{v})$ with probability 1 .

Next, we study the limiting distribution for the estimator $\widehat{\gamma}$. Given $-1 / 2<\tau<1 / 2, \widehat{\gamma}$ is a consistent estimator with convergence rate $a_{n}$, thus, we could focus on its asymptotic behavior in the neighborhood of the true thresholds. Let $\gamma=\gamma_{0}+\frac{\omega}{a_{n}}$. By the definition of $\widehat{\gamma}_{n}$, we have

$$
a_{n}\left(\widehat{\gamma}_{n}-\gamma_{0}\right)=\omega^{*}=\arg \min _{\omega \in(-\infty, \infty)}\left(S S R_{n}^{*}\left(\gamma_{0}+\frac{\omega}{a_{n}}\right)-S S R_{n}^{*}\left(\gamma_{0}\right)\right)
$$

By Equation (A.2), we know $S S R_{n}^{*}\left(\gamma_{0}+\frac{\omega}{a_{n}}\right)-S S R_{n}^{*}\left(\gamma_{0}\right)=R_{1}-R_{2}+R_{3}+R_{4}+R_{5}$, with $\gamma$ replaced by $\gamma_{0}+\frac{\omega}{a_{n}}$. Next, we turn to study the limiting behavior for each term. We only provide the proof for the case where $\omega>0$, as the proof for the case with $\omega \leq 0$ is analogous. Given $\omega>0$, we have

$$
\begin{aligned}
R_{1}= & \delta_{n}^{\prime} \sum_{t=1}^{n}\left(x_{t}\left(\gamma_{0}+\frac{\omega}{a_{n}}\right)-x_{t}\left(\gamma_{0}\right)\right)\left(x_{t}\left(\gamma_{0}+\frac{\omega}{a_{n}}\right)-x_{t}\left(\gamma_{0}\right)\right)^{\prime} \delta_{n} \\
= & n^{1-2 \tau} \delta_{0}^{\prime} n^{-2} \sum_{t=1}^{n}\left(x_{t}\left(\gamma_{0}+\frac{\omega}{a_{n}}\right)-x_{t}\left(\gamma_{0}\right)\right)\left(x_{t}\left(\gamma_{0}+\frac{\omega}{a_{n}}\right)-x_{t}\left(\gamma_{0}\right)\right)^{\prime} \delta_{0} \\
= & n^{1-2 \tau} \delta_{0}^{\prime}\left(F\left(\gamma_{0}+\frac{\omega}{a_{n}}\right)-F\left(\gamma_{0}\right)\right)\left(\int \mathbf{B}_{v}(s) \mathbf{B}_{v}^{\prime}(s) d s\right) \delta_{0}+o_{p}(1) \\
& \stackrel{p}{\rightarrow} f\left(\gamma_{0}\right) \omega \delta_{0}^{\prime}\left(\int \mathbf{B}_{v}(s) \mathbf{B}_{v}^{\prime}(s) d s\right) \delta_{0} .
\end{aligned}
$$

Note that the last equation uses the first-order Taylor expansion of $F\left(\gamma_{0}+\frac{\omega}{a_{n}}\right)$ around $\gamma_{0}$. For $R_{2}$, we have

$$
\begin{aligned}
R_{2} & =-2 \sum_{t=1}^{n} \delta_{n}^{\prime}\left(x_{t}\left(\gamma_{0}+\frac{\omega}{a_{n}}\right)-x_{t}\left(\gamma_{0}\right)\right) u_{t}=-2 n^{1 / 2-\tau} \delta_{0}^{\prime} \frac{1}{n} \sum_{t=1}^{n}\left(x_{t}\left(\gamma_{0}+\frac{\omega}{a_{n}}\right)-x_{t}\left(\gamma_{0}\right)\right) u_{t} \\
& \Rightarrow-2 \sqrt{a_{n}} \delta_{0}^{\prime} \sigma_{u} \int \mathbf{B}_{v}(s) d\left(W\left(s, F\left(\gamma_{0}+\frac{\omega}{a_{n}}\right)\right)-W\left(s, F\left(\gamma_{0}\right)\right)\right) \\
& =-2 \sqrt{a_{n}} \delta_{0}^{\prime} \sigma_{u}\left(\mathbf{J}_{1}\left(F\left(\gamma_{0}+\frac{\omega}{a_{n}}\right)\right)-\mathbf{J}_{1}\left(F\left(\gamma_{0}\right)\right)\right) .
\end{aligned}
$$

Note that $\mathbf{J}_{1}(\mu)=\int \mathbf{B}_{v}(s) d W(s, \mu)$ is a mean-zero Gaussian process with an almost surely continuous sample path and with the covariance kernel $\mathbb{E}\left(\mathbf{J}_{1}\left(\mu_{1}\right) \mathbf{J}_{1}\left(\mu_{2}\right)\right)=\left(\mu_{1} \wedge \mu_{2}\right) \int \mathbf{B}_{v}(s) \mathbf{B}_{v}^{\prime}(s) d s$. Thus, $D(\omega)=$ 
$\sqrt{a_{n}} \delta_{0}^{\prime} \sigma_{u}\left(\mathbf{J}_{1}\left(F\left(\gamma_{0}+\frac{\omega}{a_{n}}\right)\right)-\mathbf{J}_{1}\left(F\left(\gamma_{0}\right)\right)\right)$ is also a Brownian motion with

$$
\operatorname{Var}(D(\omega))=\sigma_{u}^{2} a_{n}\left(F\left(\gamma_{0}+\frac{\omega}{a_{n}}\right)-F\left(\gamma_{0}\right)\right) \delta_{0}^{\prime} \int \mathbf{B}_{v}(s) \mathbf{B}_{v}^{\prime}(s) d s \delta_{0}=\sigma_{u}^{2} f_{0} \omega \int \mathbf{B}_{v}(s) \mathbf{B}_{v}^{\prime}(s) d s+o_{p}(1) .
$$

By Equations (A.4) to (A.6), we have $R_{3}+R_{4}+R_{5}=\omega o_{p}(1)=o_{p}(1)$. Combining the convergence results of Equations (A.7) and (A.8), we have

$$
\begin{aligned}
S S R_{n}^{*}(\gamma)-S S R_{n}^{*}\left(\gamma_{0}\right) & \Rightarrow f_{0} \omega \delta_{0}^{\prime} \int \mathbf{B}_{v}(s) \mathbf{B}_{v}^{\prime}(s) d s \delta_{0}-2 D(\omega) \\
& =f_{0} \omega \delta_{0}^{\prime} \int \mathbf{B}_{v}(s) \mathbf{B}_{v}^{\prime}(s) d s \delta_{0}-2 \sigma_{u} \sqrt{f_{0} \omega \delta_{0}^{\prime} \int \mathbf{B}_{v}(s) \mathbf{B}_{v}^{\prime}(s) d s \delta_{0} \Lambda_{1}(\omega),}
\end{aligned}
$$

where $\Lambda_{1}(\omega)$ is a standard Brownian motion on $[0, \infty)$. Making the change of variables as follows

$$
\omega=\frac{\sigma_{u}^{2}}{\delta_{0}^{\prime}\left(\int \mathbf{B}_{v}(s) \mathbf{B}_{v}^{\prime}(s) d s\right) \delta_{0} f_{0}} r
$$

we have

$$
S S R_{n}^{*}(\gamma)-S S R_{n}^{*}\left(\gamma_{0}\right) \Rightarrow 2 \sigma_{u}^{2}\left(\frac{r}{2}-\Lambda_{1}(r)\right)
$$

Using continuous mapping theorem, the asymptotic distribution of $\widehat{\gamma}$ can be expressed as

$$
n^{1-2 \tau} \lambda\left(\widehat{\gamma}-\gamma_{0}\right)=r^{*} \Rightarrow \arg \max _{r \in(0, \infty)}\left(\Lambda_{1}(r)-\frac{1}{2}|r|\right) .
$$

Lastly, we establish the limiting results for $\widehat{\gamma}_{n}$ when $\tau=1 / 2$. By the definition of $\widehat{\gamma}_{n}$, we have $\widehat{\gamma}_{n}=$ $\arg \min _{\gamma \in[\underline{\gamma}, \bar{\gamma}]} S S R_{n}(\gamma)=\arg \max _{\gamma \in \underline{[}, \bar{\gamma}]}\left(S S R_{n}-S S R_{n}(\gamma)\right)$, where $S S R_{n}$ is defined as the sum of squared residuals by regressing $y_{t}$ to $x_{t}$. After some standard algebra, we have $S S R_{n}-S S R_{n}(\gamma)=Y^{\prime}\left(I-P_{n}\right) X(\gamma)\left(X^{\prime}(\gamma)(I-\right.$ $\left.\left.P_{n}\right) X(\gamma)\right)^{-1} X^{\prime}(\gamma)\left(I-P_{n}\right) Y$, where $X(\gamma)=\left(x_{1}(\gamma), x_{2}(\gamma), \ldots, x_{n}(\gamma)\right)^{\prime}$ and $X=\left(x_{1}, x_{2}, \ldots, x_{n}\right)^{\prime} . P_{n}=X\left(X^{\prime} X\right)^{-1} X^{\prime}$ is the projection matrix of $X$. Define $\Gamma_{n}(\gamma)=n^{-1} X^{\prime}(\gamma)\left(I-P_{n}\right) Y$.

Given $\tau=1 / 2$, we have $n \delta_{n}=\delta_{0}$. By Lemma A.3, it can be shown that

$$
\Gamma_{n}(\gamma) \Rightarrow \sigma_{u} \int \mathbf{B}_{v}(s) d(W(s, F(\gamma))-F(\gamma) W(s))+\left(F\left(\gamma \wedge \gamma_{0}\right)-F(\gamma) F\left(\gamma_{0}\right)\right)\left(\int \mathbf{B}_{v}(s) \mathbf{B}_{v}^{\prime}(s) d s\right) \delta_{0} \equiv \Gamma_{1}(\gamma)
$$

and $n^{-2} X^{\prime}(\gamma)\left(I-P_{n}\right) X(\gamma) \Rightarrow F(\gamma)(1-F(\gamma)) \int \mathbf{B}_{v}(s) \mathbf{B}_{v}^{\prime}(s) d s$. By continuous mapping theorem, we can show that $\left(S S R_{n}-S S R_{n}(\gamma)\right)=\Gamma_{n}^{\prime}(\gamma)\left(X^{\prime}(\gamma)\left(I-P_{n}\right) X(\gamma) / n^{2}\right)^{-1} \Gamma_{n}(\gamma) \Rightarrow \frac{1}{F(\gamma)(1-F(\gamma))} \Gamma_{1}^{\prime}(\gamma)\left(\int \mathbf{B}_{v}(s) \mathbf{B}_{v}^{\prime}(s) d s\right)^{-1} \Gamma_{1}(\gamma)$. 
Proof of Theorem 3.2: Note that

$$
\begin{aligned}
T_{n}(\gamma) & =\widehat{\delta}_{n}(\gamma)^{\prime}\left(X^{\prime}(\gamma)\left(I-P_{n}\right) X(\gamma)\right) \widehat{\delta}_{n}(\gamma) / \widehat{\sigma}_{u}^{2} \\
& =\left(I-P_{n}\right) Y^{\prime} X(\gamma)\left(X^{\prime}(\gamma)\left(I-P_{n}\right) X(\gamma)\right)^{-1} X^{\prime}(\gamma)\left(I-P_{n}\right) Y / \widehat{\sigma}_{u}^{2} \\
& =\Gamma_{n}(\gamma)^{\prime}\left(n^{-2} X(\gamma)^{\prime} X(\gamma)-n^{-2} X(\gamma)^{\prime} X\left(X^{\prime} X\right)^{-1} X X(\gamma)\right)^{-1} \Gamma_{n}(\gamma) / \widehat{\sigma}_{u}^{2}
\end{aligned}
$$

By Lemma A.3, we have $n^{-2} X(\gamma)^{\prime} X(\gamma)-n^{-2} X(\gamma)^{\prime} X\left(X^{\prime} X\right)^{-1} X X(\gamma) \stackrel{p}{\rightarrow}\left(F(\gamma)-F(\gamma)^{2}\right) \int \mathbf{B}_{v}(s) \mathbf{B}_{v}^{\prime}(s) d s$. Next, we consider the limiting result for $\Gamma_{n}(\gamma)$ for different $\tau$. When $-1 / 2<\tau<1 / 2$, we have

$$
\begin{aligned}
\Gamma_{n}(\gamma) & =\frac{1}{n} X^{\prime}(\gamma) u-\frac{1}{n} X^{\prime}(\gamma) X\left(X^{\prime} X\right)^{-1} X^{\prime} u+\frac{1}{n} X^{\prime}(\gamma) X\left(\gamma_{0}\right) \delta_{n}-\frac{1}{n} X^{\prime}(\gamma) X\left(X^{\prime} X\right)^{-1} X^{\prime} X\left(\gamma_{0}\right) \delta_{n} \\
& \Rightarrow \sigma_{u} \int_{0}^{1} \mathbf{B}_{v}(s) d(W(s, F(\gamma))-F(\gamma) W(s))+n^{1 / 2-\tau}\left(F\left(\gamma \wedge \gamma_{0}\right)-F(\gamma) F\left(\gamma_{0}\right)\right)\left(\int \mathbf{B}_{v}(s) \mathbf{B}_{v}^{\prime}(s) d s\right) \delta_{0} \\
& =O_{p}\left(n^{1 / 2-\tau}\right) \stackrel{p}{\rightarrow} \infty .
\end{aligned}
$$

It follows that $T_{n}(\gamma)=O_{p}\left(n^{1-2 \tau}\right) \stackrel{p}{\rightarrow} \infty$.

When $\tau=1 / 2$, we have

$$
\begin{aligned}
\Gamma_{n}(\gamma) & =\frac{1}{n} X^{\prime}(\gamma) u-\frac{1}{n} X^{\prime}(\gamma) X\left(X^{\prime} X\right)^{-1} X^{\prime} u+\frac{1}{n} X^{\prime}(\gamma) X\left(\gamma_{0}\right) \delta_{n}-\frac{1}{n} X^{\prime}(\gamma) X\left(X^{\prime} X\right)^{-1} X^{\prime} X\left(\gamma_{0}\right) \delta_{n} \\
& \Rightarrow \Gamma(\gamma)+\left(F\left(\gamma \wedge \gamma_{0}\right)-F(\gamma) F\left(\gamma_{0}\right)\right)\left(\int \mathbf{B}_{v}(s) \mathbf{B}_{v}^{\prime}(s) d s\right) \delta_{0}=\Gamma_{1}(\gamma) .
\end{aligned}
$$

It follows that $T_{n}(\gamma) \Rightarrow \frac{1}{\sigma_{u}^{2} F(\gamma)(1-F(\gamma))} \Gamma_{1}^{\prime}(\gamma)\left(\int \mathbf{B}_{v}(s) \mathbf{B}_{v}^{\prime}(s) d s\right)^{-1} \Gamma_{1}(\gamma)$.

Proof of Theorem 3.3: We prove the equality by showing the following two inequalities: $A s y S Z_{\gamma}(a) \geq 1-a$ and $A \operatorname{syS} Z_{\gamma}(a) \leq 1-a$ hold simultaneously. We first consider the proof of $A s y S Z_{\gamma}(a) \geq 1-a$.

By the definition of $A s y S Z_{\gamma}(a)$ as Equation (18), we can find a parameter sequence $\left(\delta_{n}, \gamma_{n}\right)$ such that $\operatorname{AsyS} Z_{\gamma}(a)=\liminf _{n \rightarrow \infty} \operatorname{Pr}_{\left(\delta_{n}, \gamma_{n}\right)}\left(\gamma_{n} \in C I_{\gamma, n}(a)\right)$. Let $\left\{b_{n}\right\}$ be a subsequence of $\{n\}$ such that $A s y S Z_{\gamma}(a)=$ $\lim _{n \rightarrow \infty} \operatorname{Pr}_{\left(\delta_{b_{n}}, \gamma_{b_{n}}\right)}\left(\gamma_{b_{n}} \in C I_{\gamma, b_{n}}(a)\right)$. Denote $\delta_{0}^{*}=n \delta_{n}$ where $\delta_{0}^{*} \in R \cup\{-\infty, \infty\}$. Because the Euclidean space is complete, we can always find a subsequence $\left\{c_{n}\right\}$ of $\left\{b_{n}\right\}$ such that $\left(c_{n} \delta_{c_{n}}, \gamma_{c_{n}}\right) \rightarrow\left(\delta_{0}^{*}, \gamma_{0}\right)$.

If $\tau=1 / 2$, we have $\delta_{0}^{*}=n n^{-1 / 2-\tau} \delta_{0}=\delta_{0} \in R$. By Theorem 3.2, we have $T_{n}=O_{p}\left(n^{1-2 \tau}\right)=O_{p}(1)<\kappa_{n}$ with probability one. Thus, $C I_{\gamma, n}(a)=C I_{\gamma, n}^{W}(a)$, and $A \operatorname{sy} S Z_{\gamma}(a)=\lim _{n \rightarrow \infty} \operatorname{Pr}_{\left(\delta_{c_{n}}, \gamma_{c_{n}}\right)}\left(\gamma_{c_{n}} \in C I_{\gamma, c_{n}}(a)\right)=$ $\lim _{n \rightarrow \infty} \operatorname{Pr}_{\left(\delta_{c_{n}}, \gamma_{c_{n}}\right)}\left(\left|\widehat{\gamma}_{c_{n}}-\gamma_{c_{n}}\right| \leq \widehat{q}_{\gamma, 1-a}^{W}\right) \geq \lim _{n \rightarrow \infty} \operatorname{Pr}_{\left(\delta_{c_{n}}, \gamma_{c_{n}}\right)}\left(\left|\widehat{\gamma}_{c_{n}}-\gamma_{c_{n}}\right| \leq \widehat{q}_{\gamma, 1-a}^{W}\left(\gamma_{0}, \delta_{0}\right)\right)=1-a$. The inequality uses the fact that $\widehat{q}_{\gamma, 1-a}^{W}=\sup _{\gamma \in[\underline{\gamma}, \bar{\gamma}]} \sup _{\delta \in R} q_{\gamma, 1-a}^{W}(\gamma, \delta)$. The last equation uses the fact that $\left|\widehat{\gamma}_{c_{n}}-\gamma_{c_{n}}\right|$ converges to $\left|\widehat{\gamma}\left(\gamma_{0}, \delta_{0}\right)-\gamma_{0}\right|$ and $\widehat{q}_{\gamma, 1-a}^{W}\left(\gamma_{0}, \delta_{0}\right)$ is defined as the $(1-a)$ quantile of the limiting distribution of $\left|\widehat{\gamma}\left(\gamma_{0}, \delta_{0}\right)-\gamma_{0}\right|$.

If $-1 / 2<\tau<1 / 2, \delta_{0}^{*}=n n^{-1 / 2-\tau} \delta_{0}=n^{1 / 2-\tau} \delta_{0}= \pm \infty$. Note that $n^{-\nu} \kappa_{n}^{1 / 2} \rightarrow 0$ for any $\nu>0$, we have $T_{n}=O_{p}\left(n^{1-2 \tau}\right)>\kappa_{n}$ with probability approaching one. Thus, $C I_{\gamma, c_{n}}(a)=C I_{\gamma, c_{n}}^{I}(a)$ and $\operatorname{AsyS}_{\gamma}(a)=$ $\lim _{n \rightarrow \infty} \operatorname{Pr}_{\left(\delta_{c_{n}}, \gamma_{c_{n}}\right)}\left(\gamma_{c_{n}} \in C I_{\gamma, c_{n}}(a)\right)=\lim _{n \rightarrow \infty} \operatorname{Pr}\left(\gamma_{c_{n}} \in C I_{\gamma, c_{n}}^{I}(a)\right)=\lim _{n \rightarrow \infty} \operatorname{Pr}\left(L R\left(\gamma_{c_{n}}\right) \leq q_{1-\alpha}^{I}\right)$. As 
$L R_{n}\left(\gamma_{n}\right) \Rightarrow \sup _{r \in(-\infty, \infty)}(2 \Lambda(r)-|r|), L R_{c_{n}}\left(\gamma_{c_{n}}\right)$ converges to $\sup _{r \in(-\infty, \infty)}(2 \Lambda(r)-|r|)$ and $q_{1-\alpha}^{I}$ is the $1-a$ quantile of $\sup _{r \in(-\infty, \infty)}(2 \Lambda(r)-|r|)$. Thus, $A s y S Z_{\gamma}(a)=\lim _{n \rightarrow \infty} \operatorname{Pr}\left(L R\left(\gamma_{c_{n}}\right) \leq q_{1-\alpha}^{I}\right) \geq 1-a$.

Next, we consider the other side $\operatorname{AsyS} Z_{\gamma}(a) \leq 1-a$. Let $\delta_{n}=n^{-1 / 2} \delta_{0}$ and $\gamma_{n}=\gamma_{0}$ with $\delta_{0} \in R /\{0\}$. By definition, we have $\operatorname{AsyS} Z_{\gamma}(a) \leq \liminf _{n \rightarrow \infty} \operatorname{Pr}_{\left(\delta_{0}, \gamma_{0}\right)}\left(\gamma_{0} \in C I_{\gamma, n}(a)\right)$. When $\delta_{n}=n^{-1 / 2} \delta_{0}$, we have $T_{n}=O_{p}\left(n^{1-2 \tau}\right)=$ $O_{p}(n)>\kappa_{n}$ with probability approaching one. Thus, $C I_{\gamma, n}(a)=C I_{\gamma, n}^{I}(a)$, and $\liminf \operatorname{in}_{n \rightarrow \infty} \operatorname{Pr}_{\left(\delta_{n}, \gamma_{0}\right)}\left(\gamma_{0} \in\right.$ $\left.C I_{\gamma, n}(a)\right)=\liminf _{n \rightarrow \infty} \operatorname{Pr}\left(\gamma_{0} \in C I_{\gamma, n}^{I}(a)\right)=\liminf _{n \rightarrow \infty} \operatorname{Pr}\left(L R_{n}\left(\gamma_{0}\right) \leq q_{1-\alpha}^{I}\right)=1-a$ where the last equality holds because $L R_{n}\left(\gamma_{0}\right) \Rightarrow \sup _{r \in(-\infty, \infty)}(2 \Lambda(r)-|r|)$.

\section{Part B}

Define the moment functionals for the stationary regressors $z_{t}$ as

$$
\begin{aligned}
h(\gamma) & =\mathbb{E}\left(z_{t} I\left(q_{t} \leq \gamma\right)\right), h_{1}(\gamma)=\mathbb{E}\left(z_{t} I\left(q_{t-1} \leq \gamma\right)\right), h_{2}(\gamma)=\mathbb{E}\left(z_{t-1} I\left(q_{t} \leq \gamma\right)\right), \\
H & =\mathbb{E}\left(z_{t} z_{t}^{\prime}\right), H_{1}=\mathbb{E}\left(z_{t} z_{t-1}^{\prime}\right) .
\end{aligned}
$$

LEMMA B.1. Under Assumptions (4.1), (4.2), (2.3) and (2.4), for any $\gamma \in[\underline{\gamma}, \bar{\gamma}]$, as $n \rightarrow \infty$,

a) $n^{-3 / 2} \sum_{t=1}^{n} z_{t} x_{t}^{\prime}(\gamma) \stackrel{p}{\rightarrow} h(\gamma) \int \mathbf{B}_{v}^{\prime}(s) d s$,

b) $n^{-3 / 2} \sum_{t=1}^{n} z_{t} x_{t-1}^{\prime}(\gamma) \stackrel{p}{\rightarrow} h_{1}(\gamma) \int \mathbf{B}_{v}^{\prime}(s) d s$,

c) $n^{-3 / 2} \sum_{t=1}^{n} z_{t-1} x_{t}^{\prime}(\gamma) \stackrel{p}{\rightarrow} h_{2}(\gamma) \int^{\prime} \mathbf{B}_{v}^{\prime}(s) d s$,

d) $n^{-1} \sum_{t=1}^{n} z_{t} z_{t}^{\prime} \stackrel{p}{\rightarrow} H$

e) $n^{-1} \sum_{t=1}^{n} z_{t} z_{t-1}^{\prime} \stackrel{p}{\rightarrow} H_{1}$.

LEMMA B.2. Under Assumptions (4.1), (4.2), (2.3) and (2.4), for any $\gamma \in[\underline{\gamma}, \bar{\gamma}]$ and $\mu=F(\gamma)$ as $n \rightarrow \infty$,

a) $: n^{-1 / 2} \sum_{t=1}^{n} I\left(q_{t} \leq \gamma\right) \varepsilon_{t} \Rightarrow \sigma W(s, \mu)$,

b) : $n^{-1 / 2} \sum_{t=1}^{n} I\left(q_{t-1} \leq \gamma\right) \varepsilon_{t} \Rightarrow \sigma W_{1}(s, \mu)$

c) $: n^{-1} \sum_{t=1}^{n} x_{t}(\gamma)^{\prime} \varepsilon_{t} \Rightarrow \sigma \int \mathbf{B}_{v}^{\prime}(s) d W(s, \mu)$,

d) $: n^{-1} \sum_{t=1}^{n} x_{t-1}(\gamma)^{\prime} \varepsilon_{t} \Rightarrow \sigma \int \mathbf{B}_{v}^{\prime}(s) d W_{1}(s, \mu)$,

e) $: n^{-1 / 2} \sum_{t=1}^{n} z_{t} \varepsilon_{t} \Rightarrow \sigma J_{2}$

f) : $n^{-1 / 2} \sum_{t=1}^{n} z_{t-1} \varepsilon_{t} \Rightarrow \sigma J_{3}$,

where $J_{2}$ and $J_{3}$ are Gaussian random variable with mean zero and variance $H . W(s, \mu)$ and $W_{1}(s, \mu)$ are two two-parameter Brownian motions.

Proof of Lemmas B.1-B.2: The proofs are standard and are skipped for space considerations.

LEMMA B.3. If $-1 / 2<\tau<1 / 2$, under Assumptions (4.1), (4.2), (2.3) and (2.4), we have $\widehat{\gamma}_{n} \stackrel{p}{\rightarrow} \gamma_{0}$ for any $\rho \in(-1,1]$.

Proof: We conduct the proof by considering two cases according to the value of $\rho$. When $\rho<1, u_{t}=\beta^{\prime} z_{t}+\eta_{t}$ is stationary process. The proof is similar to Lemma A.5 and is skipped for brevity. When $\rho=1, u_{t}=\beta^{\prime} z_{t}+\eta_{t}$ is 
nonstationary since $\eta_{t}$ is a unit root process. Using a similar argument of Lemma A.5, we only need to show that $n^{-1+2 \tau}\left(S S R_{n}(\gamma)-S S R_{n}\left(\gamma_{0}\right)\right)$ uniformly converges to a function $b(\gamma)$ which is positive when $\gamma \in B(\epsilon)$, where $B(\epsilon)=\left\{\gamma:\left|\gamma-\gamma_{0}\right|>\varepsilon\right\}$ for any $\varepsilon>0$. Note that Equation (A.1) still holds, i.e., $n^{-1+2 \tau}\left(\operatorname{SSR}_{n}(\gamma)-\operatorname{SSR}_{n}\left(\gamma_{0}\right)\right)=$ $S_{1}+S_{2}+S_{3}$. Next, we will show that both $S_{2}$ and $S_{3}$ converge to zero but $S_{1}$ uniformly converges a function $b(\gamma)$ which is positive when $\gamma \in B(\epsilon)$.

First, note that the stationary component $\beta^{\prime} z_{t}$ of $u_{t}$ is asymptotically negligible, implying that $S_{3}=$ $n^{-1+2 \tau}\left(u^{\prime}\left(I-P_{\gamma}^{*}\right) u-u^{\prime}\left(I-P_{\gamma_{0}}^{*}\right) u\right)=n^{-1+2 \tau}\left(\eta^{\prime} P_{\gamma}^{*} \eta-\eta^{\prime} P_{\gamma_{0}}^{*} \eta\right)+o_{p}(1)$. Denote $B_{\eta}(s)$ as a Brownian motion such that $\frac{1}{\sqrt{n}} \eta_{[n s]} \stackrel{a . s .}{\rightarrow} B_{\eta}(s)$. By Lemma B.1, we have

$$
n^{-2} \eta^{\prime} X^{*}(\gamma)\left(X^{*}(\gamma)^{\prime} X^{*}(\gamma)\right)^{-1} X^{*}(\gamma)^{\prime} \eta \stackrel{a . s .}{\rightarrow}\left(\int \mathbf{B}_{v}(s) B_{\eta}(s) d s\right)^{\prime}\left(\int \mathbf{B}_{v}(s) \mathbf{B}_{v}^{\prime}(s) d s\right)^{-1}\left(\int \mathbf{B}_{v}(s) B_{\eta}(s) d s\right)
$$

which is unrelated to $\gamma$. Thus, $\eta^{\prime} P_{\gamma}^{*} \eta-\eta^{\prime} P_{\gamma_{0}}^{*} \eta=o_{p}(1)$ and it follows that $S_{3}=n^{-1+2 \tau}\left(\eta^{\prime} P_{\gamma}^{*} \eta-\eta^{\prime} P_{\gamma_{0}}^{*} \eta\right)+o_{p}(1)=$ $o_{p}(1)$.

To prove $S_{2}$ converge to zero almost surely, we first consider the case where $\gamma>\gamma_{0}$. For the case where $\gamma \leq \gamma_{0}$, the proof is similar and not repeated here. Given $\gamma>\gamma_{0}$, we have

$$
\begin{aligned}
& n^{-2} X^{\prime}(\gamma) X\left(\gamma_{0}\right)=n^{-2} X^{\prime}\left(\gamma_{0}\right) X\left(\gamma_{0}\right) \stackrel{p}{\rightarrow} F\left(\gamma_{0}\right) \int \mathbf{B}_{v}(s) \mathbf{B}_{v}^{\prime}(s) d s, \\
& n^{-2} X\left(\gamma_{0}\right)^{\prime}(X-X(\gamma))=0 \\
& n^{-2}\left(X-X\left(\gamma_{0}\right)\right)^{\prime} X(\gamma) \stackrel{p}{\rightarrow}\left(F(\gamma)-F\left(\gamma_{0}\right)\right) \int \mathbf{B}_{v}(s) \mathbf{B}_{v}^{\prime}(s) d s .
\end{aligned}
$$

It follows that

$$
n^{-2} X^{*}\left(\gamma_{0}\right)^{\prime} X^{*}(\gamma) \stackrel{p}{\rightarrow}\left(\begin{array}{c}
F\left(\gamma_{0}\right) \int \mathbf{B}_{v}(s) \mathbf{B}_{v}^{\prime}(s) d s, 0 \\
\left(F(\gamma)-F\left(\gamma_{0}\right)\right) \int \mathbf{B}_{v}(s) \mathbf{B}_{v}^{\prime}(s) d s,(1-F(\gamma)) \int \mathbf{B}_{v}(s) \mathbf{B}_{v}^{\prime}(s) d s
\end{array}\right) .
$$

Using the above convergence results, it can be shown that

$$
n^{-1+2 \tau} \delta_{n}^{\prime} X\left(\gamma_{0}\right)^{\prime} \eta=n^{1 / 2+\tau} \delta_{0}^{\prime} n^{-2} \sum_{t=1}^{n} x_{t}\left(\gamma_{0}\right) \eta \stackrel{p}{\rightarrow} n^{1 / 2+\tau} \delta_{0}^{\prime}\left(\begin{array}{c}
F\left(\gamma_{0}\right) \int \mathbf{B}_{v}(s) B_{\eta}(s) d s \\
\left(1-F\left(\gamma_{0}\right)\right) \int \mathbf{B}_{v}(s) B_{\eta}(s) d s
\end{array}\right)
$$

and

$$
n^{-1+2 \tau} \delta_{n}^{\prime} X\left(\gamma_{0}\right)^{\prime} P_{\gamma}^{*} \eta \stackrel{p}{\rightarrow} n^{1 / 2+\tau} \delta_{0}^{\prime}\left(\begin{array}{c}
F\left(\gamma_{0}\right) \int \mathbf{B}_{v}(s) B_{\eta}(s) d s \\
\left(1-F\left(\gamma_{0}\right)\right) \int \mathbf{B}_{v}(s) B_{\eta}(s) d s
\end{array}\right)
$$

Thus, $S_{2}=2 n^{-1+2 \tau} \delta_{n}^{\prime} X\left(\gamma_{0}\right)^{\prime}\left(I-P_{\gamma}^{*}\right) u=2 n^{-1+2 \tau} \delta_{n}^{\prime} X\left(\gamma_{0}\right)^{\prime} \eta-2 n^{-1+2 \tau} \delta_{n}^{\prime} X\left(\gamma_{0}\right)^{\prime} P_{\gamma}^{*} \eta+o_{p}(1)=o_{p}(1)$.

Moreover, using a similar argument in Lemma A.5, we can show $S_{1} \stackrel{p}{\rightarrow} b(\gamma)$ uniformly and $b(\gamma)$ is strictly positive when $\gamma \in B(\epsilon)$. Combining the convergence results for $S_{1}, S_{2}$ and $S_{3}$, we complete the proof. 
Proof of Theorem 4.1: Under Assumption (4.1), the model can be rewritten as $y_{t}=\alpha^{\prime} x_{t}+\delta_{n}^{\prime} x_{t}\left(\gamma_{0}\right)+u_{t}=$ $\theta_{1}^{\prime} x_{t}^{*}(\widehat{\gamma})+\eta_{t}^{*}$, where $x_{t}^{*}(\widehat{\gamma})=\left(x_{t}(\widehat{\gamma})^{\prime}, x_{t}^{\prime}-x_{t}^{\prime}(\widehat{\gamma}), z_{t}\right)^{\prime}, \theta_{1}=\left(\alpha^{\prime}+\delta_{n}^{\prime}, \alpha^{\prime}, \beta^{\prime}\right)^{\prime}$ and $\eta_{t}^{*}=\eta_{t}+\theta_{1}^{\prime}\left(x_{t}^{*}\left(\gamma_{0}\right)-x_{t}^{*}(\widehat{\gamma})\right)$. Since $\theta_{1}^{\prime}\left(x_{t}^{*}\left(\gamma_{0}\right)-x_{t}^{*}(\widehat{\gamma})\right)=\delta_{n}^{\prime}\left(x_{t}\left(\gamma_{0}\right)-x_{t}(\widehat{\gamma})\right)$, we have $\eta_{t}^{*}=\eta_{t}+\delta_{n}^{\prime}\left(x_{t}\left(\gamma_{0}\right)-x_{t}(\widehat{\gamma})\right)$. The LS residual $\eta_{t}(\widehat{\gamma})$ can be expressed as $\widehat{\eta}_{t}(\widehat{\gamma})=y_{t}-\widehat{\theta}_{1}(\widehat{\gamma})^{\prime} x_{t}^{*}(\widehat{\gamma})=\eta_{t}+\delta_{n}^{\prime}\left(x_{t}\left(\gamma_{0}\right)-x_{t}(\widehat{\gamma})\right)+\left(\widehat{\theta}_{1}(\widehat{\gamma})-\theta_{1}\right)^{\prime} x_{t}^{*}(\widehat{\gamma})$. Next, we conduct the proof by considering two cases according to the value of $\tau$.

Case 1: $-1 / 2<\tau<1 / 2$, by Lemma B.3, we have $\widehat{\gamma} \stackrel{p}{\rightarrow} \gamma_{0}$ and $\delta_{n}^{\prime}=O_{p}\left(n^{-1 / 2-\tau}\right)=o_{p}(1)$. Thus $\delta_{n}^{\prime}\left(x_{t}\left(\gamma_{0}\right)-x_{t}(\widehat{\gamma})\right)=o_{p}(1)$. If $\rho=1, \eta_{t}$ is a unit root; thus, $\widehat{\eta}_{t}(\widehat{\gamma})=\eta_{t}+\delta_{n}^{\prime}\left(x_{t}\left(\gamma_{0}\right)-x_{t}(\widehat{\gamma})\right)+\left(\widehat{\theta}_{1}\left(\widehat{\gamma}^{\prime}\right)-\theta_{1}\right)^{\prime} x_{t}^{*}(\widehat{\gamma})$ is also a unit root process and it can be shown that $\widehat{\rho}=1+O_{p}\left(\frac{1}{n}\right)$. If $\rho<1, \eta_{t}$ is stationary. Since $\widehat{\gamma} \stackrel{p}{\rightarrow} \gamma_{0}$, it can be shown that $D_{n}\left(\widehat{\theta}_{1}(\widehat{\gamma})-\theta_{1}\right)=O_{p}\left(n^{-1 / 2}\right)$, where $D_{n}=\operatorname{diag}\left\{n^{1 / 2} I_{d_{1}}, n^{1 / 2} I_{d_{1}}, I_{d_{2}}\right\}$ is a weighting matrix. It follows that $\widehat{\eta}_{t}(\widehat{\gamma})=\eta_{t}+\delta_{n}^{\prime}\left(x_{t}\left(\gamma_{0}\right)-x_{t}(\widehat{\gamma})\right)+\left(\widehat{\theta}_{1}(\widehat{\gamma})-\theta_{1}\right)^{\prime} x_{t}^{*}(\widehat{\gamma})=\eta_{t}+o_{p}(1)+\left(\widehat{\theta}_{1}(\widehat{\gamma})-\theta_{1}\right)^{\prime} D_{n} D_{n}^{-1} x_{t}^{*}(\widehat{\gamma})^{\prime} \stackrel{p}{\rightarrow} \eta_{t}$. As $\eta_{t}$ is assumed to be $\eta_{t}=\rho \eta_{t-1}+\varepsilon_{t}$, it can be shown that $\widehat{\rho}=\rho+O_{p}\left(\frac{1}{\sqrt{n}}\right)$.

Case 2: $\tau=1 / 2, \widehat{\gamma}$ is inconsistent. Noting that $n \delta_{n}=\delta_{0}$, we have $\delta_{n}^{\prime}\left(x_{t}\left(\gamma_{0}\right)-x_{t}(\widehat{\gamma})\right)=O_{p}\left(\frac{1}{\sqrt{n}}\right)=o_{p}(1)$. If $\rho=1$, the term $\widehat{\eta}_{t}(\widehat{\gamma})=\eta_{t}+\delta_{n}^{\prime}\left(x_{t}\left(\gamma_{0}\right)-x_{t}(\widehat{\gamma})\right)+\left(\widehat{\theta}_{1}(\widehat{\gamma})-\theta_{1}\right)^{\prime} x_{t}^{*}(\widehat{\gamma})$ is an I $(1)$ process and it can be shown that $\widehat{\rho}(\widehat{\gamma})=1+O_{p}\left(\frac{1}{n}\right)$. If $\rho<1$, we can show that $D_{n}\left(\widehat{\theta}_{1}(\widehat{\gamma})-\theta_{1}\right)=O_{p}\left(n^{-1 / 2}\right)$. Thus, the following convergence still holds: $\widehat{\eta}_{t}(\widehat{\gamma})=\eta_{t}+\delta_{n}^{\prime}\left(x_{t}\left(\gamma_{0}\right)-x_{t}(\widehat{\gamma})\right)+\left(\widehat{\theta}_{1}(\widehat{\gamma})-\theta_{1}\right)^{\prime} x_{t}^{*}(\widehat{\gamma}) \stackrel{p}{\rightarrow} \eta_{t}$, implying that $\widehat{\rho}=\rho+O_{p}\left(\frac{1}{\sqrt{n}}\right)$.

Lemma B.4: For any $\rho \in(-1,1]$, there exists a nonrandom weighting matrix $\widetilde{D}_{n}$ such that

(i) : $n^{-1} \widetilde{D}_{n}^{-1} \widetilde{A}_{1}(\gamma)^{\prime} \widetilde{A}_{1}(\gamma) \widetilde{D}_{n}^{-1} \stackrel{p}{\rightarrow} \widetilde{G}(\gamma)$,

(ii) $: n^{-1 / 2} \widetilde{D}_{n}^{-1} \widetilde{A}_{1}(\gamma)^{\prime} \widetilde{\eta} \Rightarrow \widetilde{\phi}(\gamma)$,

where $\widetilde{G}(\gamma)$ and $\widetilde{\phi}(\gamma)$ are defined below.

Proof: When $\rho<1$, define the weighting matrix $\widetilde{D}_{n}=\operatorname{diag}\left\{n^{1 / 2} I_{d_{1}}, n^{1 / 2} I_{d_{1}}, I_{d_{2}}\right\}$,

$$
\begin{aligned}
G(\gamma) & =\left(\begin{array}{ccc}
\int \mathbf{B}_{v}(s) \mathbf{B}_{v}^{\prime}(s) d s & F(\gamma) \int \mathbf{B}_{v}(s) \mathbf{B}_{v}^{\prime}(s) d s & 0 \\
F(\gamma) \int \mathbf{B}_{v}(s) \mathbf{B}_{v}^{\prime}(s) d s & F(\gamma) \int \mathbf{B}_{v}(s) \mathbf{B}_{v}^{\prime}(s) d s & \left(\int \mathbf{B}_{v}(s) d s\right) h(\gamma)^{\prime} \\
0 & h(\gamma)\left(\int \mathbf{B}_{v}^{\prime}(s) d s\right) & H
\end{array}\right), \\
G_{1}(\gamma) & =\left(\begin{array}{ccc}
\int \mathbf{B}_{v}(s) \mathbf{B}_{v}^{\prime}(s) d s & F(\gamma) \int \mathbf{B}_{v}(s) \mathbf{B}_{v}^{\prime}(s) d s & 0 \\
F(\gamma) \int \mathbf{B}_{v}(s) \mathbf{B}_{v}^{\prime}(s) d s & F_{1}(\gamma, \gamma) \int \mathbf{B}_{v}(s) \mathbf{B}_{v}^{\prime}(s) d s & \left(\int \mathbf{B}_{v}(s) d s\right) h_{2}^{\prime}(\gamma) \\
0 & h_{1}(\gamma)\left(\int \mathbf{B}_{v}^{\prime}(s) d s\right) & H_{1}
\end{array}\right),
\end{aligned}
$$

and

$$
\widetilde{\phi}(\gamma)=\left(\begin{array}{c}
\sigma \int \mathbf{B}_{v}(s) d \widetilde{W}(s) \\
\sigma \int \mathbf{B}_{v}(s) d \widetilde{W}(s, F(\gamma)) \\
\sigma \widetilde{J}
\end{array}\right)
$$

where $\widetilde{W}(s)=(1-\rho) W(s), \widetilde{W}(s, F(\gamma))=W(s, F(\gamma))-\rho W_{1}(s, F(\gamma))$ and $\widetilde{J}=J_{2}-\rho J_{3}$. 
By Lemmas B.1, B.2 and Theorem 4.1, it can be shown that

$$
\begin{aligned}
n^{-1} \sum_{t=1}^{n} \widetilde{D}_{n}^{-1} \widetilde{A}_{1 t}(\gamma) \widetilde{A}_{1 t}(\gamma)^{\prime} \widetilde{D}_{n}^{-1}= & n^{-1} \sum_{t=1}^{n} \widetilde{D}_{n}^{-1} A_{1 t}(\gamma) A_{1 t}(\gamma)^{\prime} \widetilde{D}_{n}^{-1}+n^{-1} \widehat{\rho}^{2} \sum_{t=1}^{n} \widetilde{D}_{n}^{-1} A_{1 t-1}(\gamma) A_{1 t-1}(\gamma)^{\prime} \widetilde{D}_{n}^{-1} \\
& -\widehat{\rho} \sum_{t=1}^{n} \widetilde{D}_{n}^{-1} A_{1 t}(\gamma) A_{1 t-1}(\gamma)^{\prime} \widetilde{D}_{n}^{-1}-\widehat{\rho} \sum_{t=1}^{n} \widetilde{D}_{n}^{-1} A_{1 t-1}(\gamma) A_{1 t}(\gamma)^{\prime} \widetilde{D}_{n}^{-1} \\
& \stackrel{p}{\rightarrow} G(\gamma)+\rho^{2} G(\gamma)-\rho\left(G_{1}(\gamma)+G_{1}^{\prime}(\gamma)\right) \equiv \widetilde{G}(\gamma) .
\end{aligned}
$$

By Lemmas B.1, B.2 and Theorem 4.1, we can also show that

$$
n^{-1 / 2} \sum_{t=1}^{n} \widetilde{D}_{n}^{-1} \widetilde{A}_{1 t}(\gamma) \widetilde{\eta}_{t}=\left(\begin{array}{c}
n^{-1} \sum_{t=1}^{n} \widetilde{x}_{t} \widetilde{\eta}_{t} \\
n^{-1} \sum_{t=1}^{n} D_{n}^{-1} \widetilde{x}_{t}(\gamma) \widetilde{\eta}_{t} \\
n^{-1 / 2} \sum_{t=1}^{n} \widetilde{z}_{t} \widetilde{\eta}_{t}
\end{array}\right) \Rightarrow \widetilde{\phi}(\gamma)
$$

When $\rho=1$, using a similar argument for the case of $\rho<1$, we can find two random matrices $\widetilde{G}(\gamma)$ and $\widetilde{\phi}(\gamma)$ and establish the convergence results.

Proof of Theorem 4.2: The proof is similar to that of Theorem 3.1 and is skipped for brevity.

Proof of Theorem 4.3: The proof is similar to that of Theorem 3.2 and is skipped for brevity. 
Table 1: The Mean Squared Errors (MSE) of Threshold Estimators

\begin{tabular}{|c|c|c|c|c|c|c|c|}
\hline & & \multicolumn{2}{|c|}{$n=100$} & \multicolumn{2}{|c|}{$n=200$} & \multicolumn{2}{|c|}{$n=400$} \\
\hline \multicolumn{8}{|c|}{ Panel A: $\tau=0$} \\
\hline$\rho$ & $\beta$ & $\operatorname{MSE}(\widehat{\gamma})$ & $\operatorname{MSE}(\widetilde{\gamma})$ & $\operatorname{MSE}(\widehat{\gamma})$ & $\operatorname{MSE}(\widetilde{\gamma})$ & $\operatorname{MSE}(\widehat{\gamma})$ & $\operatorname{MSE}(\widetilde{\gamma})$ \\
\hline 0 & 0 & .012 & .019 & .004 & .0040 & .0021 & .0022 \\
\hline 0 & .5 & .013 & .009 & .004 & .0020 & .0008 & .0003 \\
\hline 1 & 0 & .191 & .002 & .221 & .0056 & .2030 & .0002 \\
\hline 1 & .5 & .314 & .005 & .197 & .0019 & .2340 & .0002 \\
\hline .95 & 0 & .129 & .019 & .097 & .0010 & .0320 & .0001 \\
\hline .95 & .5 & .125 & .002 & .129 & .0031 & .0310 & .0004 \\
\hline-.95 & 0 & .093 & .004 & .043 & .0002 & .0270 & .0002 \\
\hline-.95 & .5 & .171 & .020 & .095 & .0031 & .0230 & .0003 \\
\hline \multicolumn{8}{|c|}{ Panel B: $\tau=0.5$} \\
\hline 0 & 0 & .365 & .379 & .485 & .4720 & .4540 & .4550 \\
\hline 0 & .5 & .519 & .509 & .518 & .4880 & .5100 & .4540 \\
\hline 1 & 0 & 1.17 & .592 & .654 & .5270 & .7170 & .4080 \\
\hline 1 & .5 & .435 & .302 & .460 & .3890 & .5440 & .4290 \\
\hline .95 & 0 & .586 & .410 & .572 & .3800 & .5220 & .4040 \\
\hline .95 & .5 & .375 & .359 & .557 & .4400 & .4940 & .3670 \\
\hline-.95 & 0 & .800 & .608 & .422 & .3640 & .6060 & .4120 \\
\hline-.95 & .5 & .742 & .530 & .532 & .4200 & .6220 & .3560 \\
\hline
\end{tabular}

Note: The model is $y_{t}=\alpha x_{t}+\delta_{n} x_{t}\left(q_{t} \leq \gamma_{0}\right)+\beta v_{t}+u_{t}$ with $x_{t}=x_{t-1}+v_{t}, u_{t}=\rho u_{t-1}+\varepsilon_{t}$ and $q_{t}=0.5 q_{t-1}+e_{t}$, where $v_{t}, \varepsilon_{t}$ and $e_{t}$ are i.i.d. $N(0,1)$. The true threshold value $\gamma_{0}$ is set as 0 and the coefficient $\alpha$ is set as 1 . The parameter $\rho$ is set as either $0,1,0.95$ or -0.95 and the parameter $\beta$ is set as 0 or 0.5 . The size of the threshold effect $\delta_{n}$ is set as $\delta_{n}=2 n^{-1 / 2-\tau}$, where $\tau$ is 0 or 0.5 . $\operatorname{MSE}(\widehat{\gamma})$ is the MSE calculated for the profiled LS estimator of $\gamma_{0}$ and $\operatorname{MSE}(\widetilde{\gamma})$ is for the FGLS estimator. The sample size $n$ takes either 100, 200 or 400. The replication number is 1000 . 
Table 2: Size Performance

\begin{tabular}{|c|c|c|c|c|c|c|c|c|c|c|c|}
\hline \multirow[b]{2}{*}{$\rho$} & \multirow[b]{2}{*}{$\delta_{n}$} & \multirow[b]{2}{*}{$\beta$} & \multicolumn{3}{|c|}{$n=100$} & \multicolumn{3}{|c|}{$n=200$} & \multicolumn{3}{|c|}{$n=400$} \\
\hline & & & $10 \%$ & $5 \%$ & $1 \%$ & $10 \%$ & $5 \%$ & $1 \%$ & $10 \%$ & $5 \%$ & $1 \%$ \\
\hline \multicolumn{12}{|c|}{ Panel A: $\mathbf{T}_{n}$} \\
\hline 0 & 0 & 0 & .078 & .028 & .005 & .114 & .056 & .018 & .098 & .046 & .008 \\
\hline 0 & 0 & .5 & .120 & .092 & .034 & .084 & .050 & .008 & .076 & .052 & .010 \\
\hline 1 & 0 & 0 & .132 & .082 & .030 & .064 & .034 & .014 & .068 & .044 & .013 \\
\hline 1 & 0 & .5 & .086 & .052 & .022 & .134 & .074 & .022 & .112 & .076 & .018 \\
\hline .95 & 0 & 0 & .128 & .073 & .042 & .178 & .072 & .030 & .104 & .046 & .008 \\
\hline .95 & 0 & .5 & .134 & .075 & .020 & .146 & .064 & .004 & .132 & .061 & .016 \\
\hline-.95 & 0 & 0 & .120 & .072 & .010 & .106 & .060 & .018 & .093 & .042 & .015 \\
\hline-.95 & 0 & .5 & .070 & .034 & .008 & .118 & .064 & .006 & .092 & .039 & .006 \\
\hline \multicolumn{12}{|c|}{ Panel B: $\widetilde{\mathbf{T}}_{n}$} \\
\hline 0 & 0 & 0 & .110 & .072 & .030 & .102 & .062 & .022 & .098 & .047 & .010 \\
\hline 0 & 0 & .5 & .144 & .084 & .028 & .076 & .044 & .014 & .088 & .046 & .008 \\
\hline 1 & 0 & 0 & .084 & .058 & .020 & .112 & .038 & .018 & .104 & .054 & .014 \\
\hline 1 & 0 & .5 & .130 & .062 & .014 & .082 & .042 & .014 & .116 & .055 & .012 \\
\hline .95 & 0 & 0 & .114 & .062 & .002 & .072 & .060 & .008 & .082 & .042 & .008 \\
\hline .95 & 0 & .5 & .128 & .064 & .032 & .132 & .076 & .020 & .094 & .037 & .008 \\
\hline-.95 & 0 & 0 & .086 & .036 & .016 & .142 & .078 & .024 & .090 & .044 & .010 \\
\hline-.95 & 0 & .5 & .116 & .066 & .022 & .089 & .046 & .016 & .091 & .058 & .015 \\
\hline
\end{tabular}

Note: The model is $y_{t}=\alpha x_{t}+\delta_{n} x_{t}\left(q_{t} \leq \gamma_{0}\right)+\beta v_{t}+u_{t}$ with $x_{t}=x_{t-1}+v_{t}, u_{t}=\rho u_{t-1}+\varepsilon_{t}$ and $q_{t}=0.5 q_{t-1}+e_{t}$, where $v_{t}, \varepsilon_{t}$ and $e_{t}$ are i.i.d. $N(0,1)$. The true threshold value $\gamma_{0}$ is set as 0 and the coefficient $\alpha$ is set as 1 . The parameter $\rho$ is set as either $0,1,0.95$ or -0.95 and the parameter $\beta$ is set as 0 or 0.5 . The size of the threshold effect $\delta_{n}$ is set as 0 to check the size performance. The sample size $n$ takes either 100, 200 or 400 . The replication number is 1000 . 


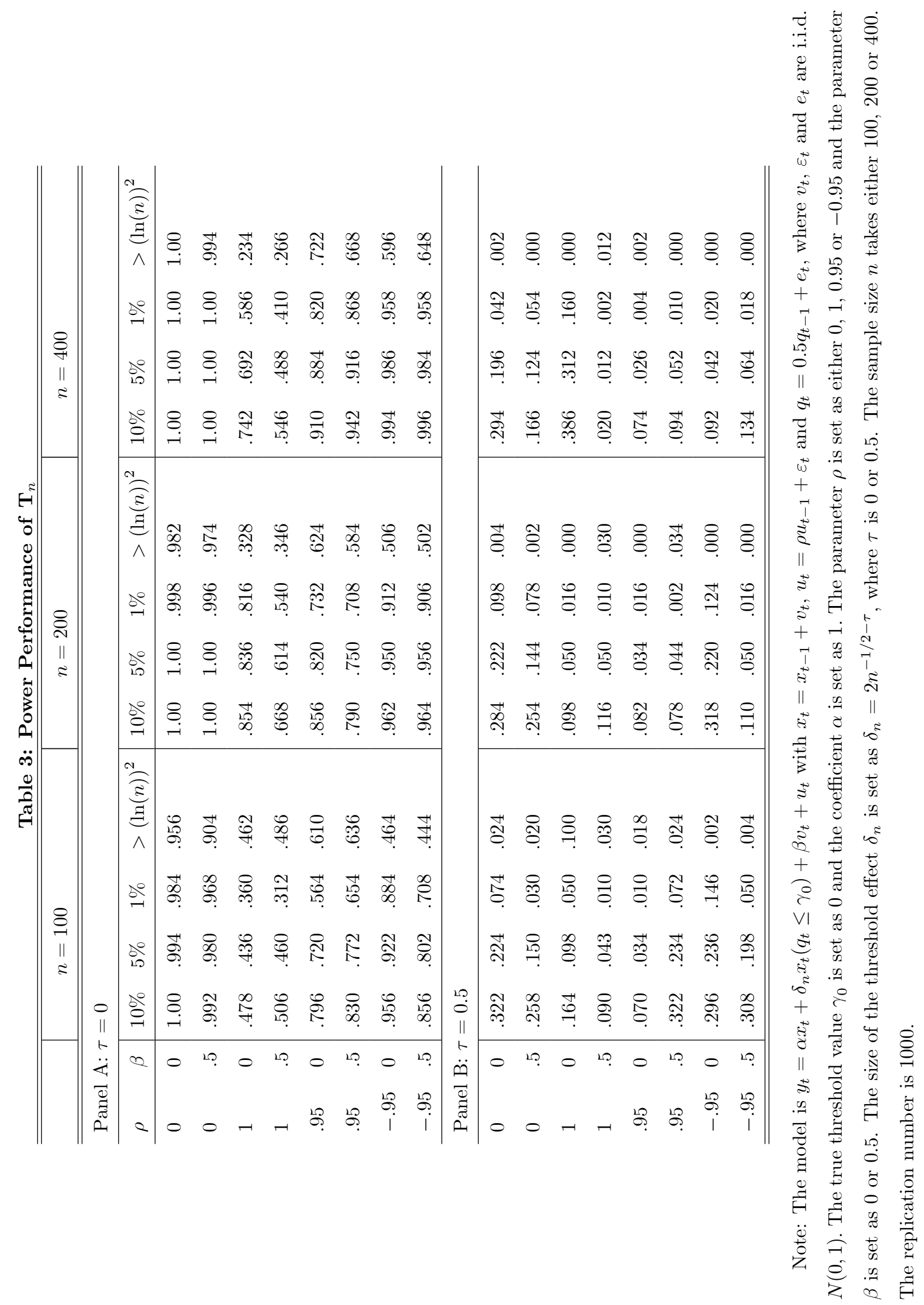




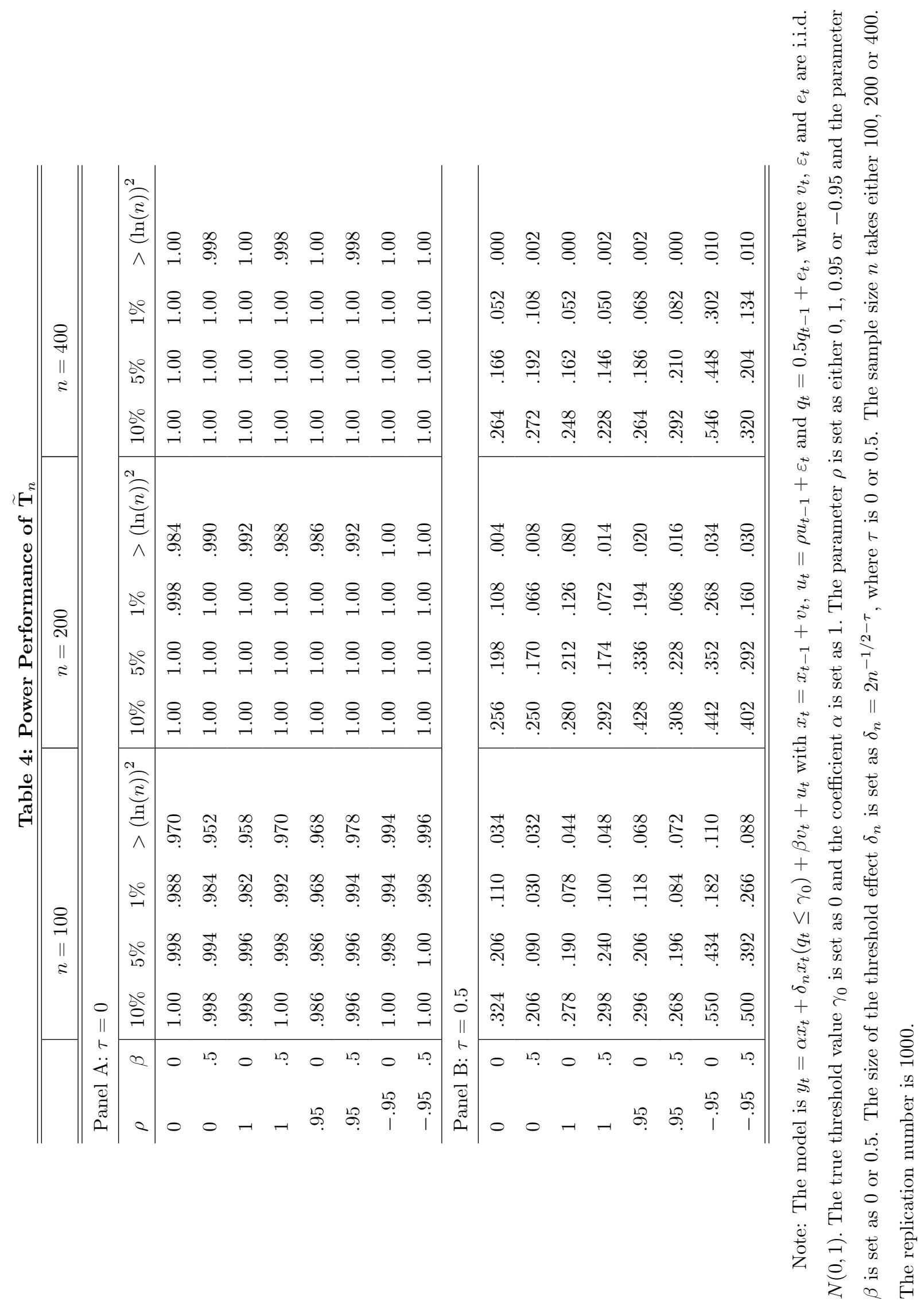

